\title{
Invariant Coupling of Determinantal Measures on Sofic Groups
}

\author{
Russell Lyons and Andreas Thom
}

\begin{abstract}
To any positive contraction $Q$ on $\ell^{2}(W)$, there is associated a determinantal probability measure $\mathbf{P}^{Q}$ on $2^{W}$, where $W$ is a denumerable set. Let $\Gamma$ be a countable sofic finitely generated group and $G=(\Gamma, \mathrm{E})$ be a Cayley graph of $\Gamma$. We show that if $Q_{1}$ and $Q_{2}$ are two $\Gamma$-equivariant positive contractions on $\ell^{2}(\Gamma)$ or on $\ell^{2}(\mathrm{E})$ with $Q_{1} \leq Q_{2}$, then there exists a $\Gamma$-invariant monotone coupling of the corresponding determinantal probability measures witnessing the stochastic domination $\mathbf{P}^{Q_{1}} \preccurlyeq \mathbf{P}^{Q_{2}}$. In particular, this applies to the wired and free uniform spanning forests, which was known before only when $\Gamma$ is residually amenable. In the case of spanning forests, we also give a second more explicit proof, which has the advantage of showing an explicit way to create the free uniform spanning forest as a limit over a sofic approximation. Another consequence of our main result is to prove that all determinantal probability measures $\mathbf{P}^{Q}$ as above are $\bar{d}$-limits of finitely dependent processes. Thus, when $\Gamma$ is amenable, $\mathbf{P}^{Q}$ is isomorphic to a Bernoulli shift, which was known before only when $\Gamma$ is abelian. We also prove analogous results for sofic unimodular random rooted graphs.
\end{abstract}

\section{Contents}

1. Introduction 2

2. Determinantal probability measures 4

3. Cayley graphs and Cayley diagrams 6

4. Tracial von Neumann algebras and their ultraproducts 10

5. Existence of invariant monotone couplings 17

6. Free uniform spanning forest measures as limits over a sofic approximation 18

7. Consequences of the invariant monotone couplings 21

8. Unimodular random rooted graphs 27

9. Existence of sofic monotone couplings 35

Acknowledgments 36

$\begin{array}{ll}\text { References } & 37\end{array}$

R.L.'s research is partially supported by NSF grant DMS-1007244 and Microsoft Research. A.T.'s research is supported by ERC-StG 277728. 


\section{Introduction}

The study of Bernoulli percolation and other random subgraphs of Cayley graphs of non-amenable groups began to flourish in the mid 1990s. Although the lack of averaging over Følner sequences was replaced by use of the Mass-Transport Principle, and expansion of all finite sets was even turned to advantage, coupling questions remained vexing: In the amenable context, if two invariant probability measures have the property that one stochastically dominates the other, then there is an invariant coupling (joining) of the two measures that witnesses the domination, which is called a monotone coupling. Whether this holds on non-amenable groups remained open until resolved in the negative by Mester [42]. Nonetheless, an invariant monotone coupling may still exist for certain pairs of measures. Indeed, the coupling question was originally motivated by the special case of the so-called wired and free spanning forest measures, denoted WSF and FSF. Bowen [9] showed that a monotone joining exists for WSF and FSF on every residually amenable group. We show that it exists for WSF and FSF on every sofic group, a wide class of groups that no group is known not to belong to. We show this as a consequence of a more general result: These spanning forest measures are examples of determinantal probability measures, where, as we review in the next section, for every positive contraction on $\ell^{2}(W), W$ being a denumerable set, there is associated a probability measure $\mathbf{P}^{Q}$ on $2^{W}$. It is known $[37,8]$ that when $Q_{1} \leq Q_{2}$, we have the stochastic domination $\mathbf{P}^{Q_{1}} \preccurlyeq \mathbf{P}^{Q_{2}}$. We show that for any pair of determinantal probability measures corresponding to equivariant positive contractions $Q_{1} \leq Q_{2}$ on $\ell^{2}$ of any sofic group, there is not only a monotone coupling of $\mathbf{P}^{Q_{1}}$ with $\mathbf{P}^{Q_{2}}$, but in fact one that is $\Gamma$-invariant. Determinantal probability measures and point processes are a class of measures that appear in a variety of contexts, including several areas of mathematics as well as physics and machine learning. See, e.g., [41, 54, 37, 30].

This is our main result, obtained via some abstract considerations of ultraproducts of tracial von Neumann algebras. The proof proceeds along the following broad outline: Given $\Gamma$-equivariant positive contractions $Q_{1} \leq Q_{2}$ and a sofic approximation to a Cayley diagram of $\Gamma$ by finite graphs $G_{n}$ labelled with the generators of $\Gamma$, we form the metric ultraproduct of the sequence of von Neumann algebras associated to the sequence $\left(G_{n}\right)_{n}$. This ultraproduct allows us to approximate $Q_{i}$ by positive contractions $Q_{1, n} \leq Q_{2, n}$ on $\ell^{2}\left(\mathrm{~V}\left(G_{n}\right)\right)$. We may then take a limit point of monotone couplings of $\mathbf{P}^{Q_{1, n}}$ with $\mathbf{P}^{Q_{2, n}}$ to obtain a $\Gamma$-invariant monotone coupling of $\mathbf{P}^{Q_{1}}$ with $\mathbf{P}^{Q_{2}}$. We remark that ultraproducts are not essential to our proofs, but they allow us to make convenient statements about operators before deducing corresponding consequences for determinantal probability measures.

We also describe some consequences for comparisons of return probabilities of random walks in random environments. While we are able to deduce a consequence for FSF that was 
not previously known, our coupling is not sufficiently explicit to deduce much more. In the motivating case of WSF and FSF, we also give a somewhat more concrete way to obtain such a coupling. In particular, this concrete approach yields a simple way to obtain the FSF as a limit over a sofic approximation. Namely, for $L \geq 0$, let $\nabla_{L}(G)$ denote the space spanned by the cycles in $G$ of length at most $L$. Write $\mathrm{FSF}_{G, L}$ for the determinantal probability measure corresponding to the orthogonal projection onto $\diamond_{L}(G)^{\perp}$. We show that if a Cayley graph $G$ is the random weak limit of $\left(G_{n}\right)_{n}$, then for $L(n) \rightarrow \infty$ sufficiently slowly, the random weak limit of $\mathrm{FSF}_{G_{n}, L(n)}$ equals $\mathrm{FSF}_{G}$. We also extend our result from the context of Cayley graphs to its natural setting of unimodular random rooted networks.

Finally, we derive a consequence of our monotone joining result for the ergodic theory of group actions. Let $\Gamma$ be a countable group and $X$ and $Y$ be two sets on which $\Gamma$ acts. A map $\phi: X \rightarrow Y$ is called $\Gamma$-equivariant if $\phi$ intertwines the actions of $\Gamma$ :

$$
\phi(\gamma x)=\gamma(\phi(x)) \quad(\gamma \in \Gamma, x \in X) .
$$

If $X$ and $Y$ are both measurable spaces, then a $\Gamma$-equivariant measurable $\phi$ is called a $\Gamma$ factor. Let $\mu$ be a measure on $X$. If $\phi$ is a $\Gamma$-factor, then the push-forward measure $\phi_{*} \mu$ is called a $\Gamma$-factor of $\mu$. The measure $\mu$ is $\Gamma$-invariant if

$$
\mu(\gamma B)=\mu(B) \quad(\gamma \in \Gamma, B \subseteq X \text { measurable }) .
$$

If $\nu$ is a measure on $Y$, then a $\nu$-a.e.-invertible $\Gamma$-factor $\phi$ such that $\nu=\phi_{*} \mu$ is called an isomorphism from $(X, \mu, \Gamma)$ to $(Y, \nu, \Gamma)$. We are interested in the case where $X$ and $Y$ are product spaces of the form $A^{\Gamma}$ or, more generally, $A^{W}$, where $A$ is a measurable space and $W$ is a countable set on which $\Gamma$ acts. In such a case, $\Gamma$ acts on $A^{W}$ by

$$
(\gamma \omega)(x):=\omega\left(\gamma^{-1} x\right) \quad\left(\omega \in A^{W}, x \in W, \gamma \in \Gamma\right) .
$$

If $\lambda$ is a probability measure on $A$ and $\mu$ is the product measure $\lambda^{W}$, then we call the action of $\Gamma$ on $A^{W}$ a Bernoulli shift. Ornstein [45] proved a number of fundamental results about Bernoulli shifts for $\Gamma=\mathbb{Z}$ that were extended in work with Weiss [46] to amenable groups. In particular, they showed that factors of Bernoulli shifts are isomorphic to Bernoulli shifts. On the other hand, in the non-amenable setting, Popa [50] gave an example of a factor of a Bernoulli shift that is not isomorphic to a Bernoulli shift. More generally, it is not well understood which actions are factors of Bernoulli shifts when $\Gamma$ is not amenable. The utility of factors of Bernoulli shifts on non-amenable groups has been shown in various ways; for example, see [50, 14, 27, 39, 1, 33, 40].

It is easy to see that every factor of a Bernoulli shift is a $\bar{d}$-limit of finitely dependent processes, by approximating the factor with a block factor. When $\Gamma$ is amenable, the converse is true: every $\bar{d}$-limit of finitely dependent processes is a factor of a Bernoulli shift. Although 
we do not know whether determinantal probability measures on $2^{\Gamma}$ arising from equivariant positive contractions are factors of Bernoulli shifts, we show here that they are $\bar{d}$-limits of finitely dependent $\Gamma$-invariant probability measures on $2^{\Gamma}$ provided $\Gamma$ is sofic; in particular, when $\Gamma$ is amenable, these determinantal measures are isomorphic to Bernoulli shifts, a result that was first shown for abelian $\Gamma$ by [38].

In Section 2, we give the relevant background on determinantal measures, including the motivating case of spanning forests. We discuss various basic notions related to groups, their Cayley graphs, their von Neumann algebras, and soficity in Section 3. A review of ultraproducts and some new results we need is in Section 4. These tools then lead quickly to a proof of our main result in Section 5. Following this, Section 6 gives the alternative proof for the spanning forest measures. Consequences of an invariant coupling are discussed in Section 7 , including the definitions of the $\bar{d}$-metric and finitely dependent processes. Tools needed for the extension to unimodular random rooted networks are given in Section 8. In fact, this generalization has the advantage that the case of measures on subsets of edges, which had to be treated separately and somewhat cumbersomely in earlier sections, here can be deduced as merely a special case. After these tools are developed, we again have a short proof of the existence of unimodular (sofic) couplings in Section 9.

\section{Determinantal probability measures}

A determinantal probability measure is one whose elementary cylinder probabilities are given by determinants. More specifically, suppose that $E$ is a finite or countable set and that $Q$ is an $E \times E$ matrix. For a subset $A \subset E$, let $Q \uparrow A$ denote the submatrix of $Q$ whose rows and columns are indexed by $A$. If $\mathfrak{S}$ is a random subset of $E$ with the property that for all finite $A \subset E$, we have

$$
\mathbf{P}[A \subset \mathfrak{S}]=\operatorname{det}(Q\lceil A)
$$

then we call $\mathrm{P}$ a determinantal probability measure. The inclusion-exclusion principle in combination with (1) determines the probability of each elementary cylinder event. Therefore, for every $Q$, there is at most one probability measure satisfying (1). Conversely, it is known (see, e.g., [37]) that there is a determinantal probability measure corresponding to $Q$ if $Q$ is the matrix of a positive contraction on $\ell^{2}(E)$ (in the standard orthonormal basis), which means that for all $u \in \ell^{2}(E)$, we have $0 \leq\langle Q u, u\rangle \leq\langle u, u\rangle$.

We identify a subset of $E$ with an element of $\{0,1\}^{E}=2^{E}$ in the usual way.

An event $\mathcal{A} \subseteq 2^{E}$ is called increasing if for all $A \in \mathcal{A}$ and all $e \in E$, we have $A \cup\{e\} \in \mathcal{A}$. Given two probability measures $\mathbf{P}^{1}, \mathbf{P}^{2}$ on $2^{E}$, we say that $\mathbf{P}^{2}$ stochastically dominates $\mathrm{P}^{1}$ and write $\mathbf{P}^{1} \preccurlyeq \mathrm{P}^{2}$ if for all increasing events $\mathcal{A}$, we have $\mathrm{P}^{1}(\mathcal{A}) \leq \mathrm{P}^{2}(\mathcal{A})$. A coupling 
of two probability measures $\mathbf{P}^{1}, \mathbf{P}^{2}$ on $2^{E}$ is a probability measure $\mu$ on $2^{E} \times 2^{E}$ whose coordinate projections are $\mathbf{P}^{1}, \mathbf{P}^{2}$. A coupling $\mu$ is called monotone if

$$
\mu\left\{\left(A_{1}, A_{2}\right) ; A_{1} \subset A_{2}\right\}=1 .
$$

By Strassen's theorem [55], stochastic domination $\mathbf{P}^{1} \preccurlyeq \mathbf{P}^{2}$ is equivalent to the existence of a monotone coupling of $\mathbf{P}^{1}$ and $\mathbf{P}^{2}$. However, even if $\mathbf{P}^{1}$ and $\mathbf{P}^{2}$ are $\Gamma$-invariant probability measures on $2^{\Gamma}$ with $\mathbf{P}^{1} \preccurlyeq \mathbf{P}^{2}$, it does not follow that there is a $\Gamma$-invariant monotone coupling of $\mathbf{P}^{1}$ and $\mathbf{P}^{2}$; see [42]. We need the following theorem; see [37] and [8].

THEOREM 2.1. Let $E$ be finite and let $Q_{1} \leq Q_{2}$ be positive contractions of $\ell^{2}(E)$. Then $\mathbf{P}^{Q_{1}} \preccurlyeq \mathbf{P}^{Q_{2}}$.

The most well-known example of a (nontrivial discrete) determinantal probability measure is that where $\mathfrak{S}$ is a uniformly chosen random spanning tree of a finite connected graph $G=(\mathrm{V}, \mathrm{E})$ with $E:=\mathrm{E}$. In this case, $Q$ is the transfer current matrix $Y$, which is defined as follows. Orient the edges of $G$ arbitrarily. Regard $G$ as an electrical network with each edge having unit conductance. Then $Y(e, f)$ is the amount of current flowing along the edge $f$ when a battery is hooked up between the endpoints of $e$ of such voltage that in the network as a whole, unit current flows from the tail of $e$ to the head of $e$. The fact that (1) holds for the uniform spanning tree is due to [13] and is called the Transfer Current Theorem. The case with $|A|=1$ was shown much earlier by [32], while the case with $|A|=2$ was first shown by [11]. Write UST $_{G}$ for the uniform spanning tree measure on $G$.

The study of the analogue on an infinite graph of a uniform spanning tree was begun by [48] at the suggestion of Lyons. Pemantle showed that if an infinite connected graph $G$ is exhausted by a sequence of finite connected subgraphs $G_{n}$, then the weak limit of the uniform spanning tree measures $\mathrm{UST}_{G_{n}}$ on $G_{n}$ exists. However, it may happen that the limit measure is not supported on trees, but on forests. This limit measure is now called the free uniform spanning forest on $G$, denoted $\mathrm{FSF}_{G}$. Considerations of electrical networks play the dominant role in the proof of existence of the limit. If $G$ is itself a tree, then this measure is trivial, namely, it is concentrated on $\{G\}$. Therefore, [26] introduced another limit that had been considered more implicitly by [48] on $\mathbb{Z}^{d}$, namely, the weak limit of the uniform spanning tree measures on $G_{n}^{*}$, where $G_{n}^{*}$ is the graph $G_{n}$ with its boundary identified to a single vertex. As [48] showed, this limit also always exists on any graph and is now called the wired uniform spanning forest, denoted $\mathrm{WSF}_{G}$.

In many cases, the free and the wired limits are the same. In particular, this is the case on all euclidean lattices such as $\mathbb{Z}^{d}$. The general question of when the free and wired uniform spanning forest measures are the same turns out to be quite interesting: The measures are the same iff there are no nonconstant harmonic Dirichlet functions on $G$ (see [6]). For a 
Cayley graph of a group $\Gamma$, this condition is equivalent to the non-vanishing of the first $\ell^{2}$-Betti number of the group, i.e., $\beta_{1}^{(2)}(\Gamma) \neq 0$. This important property of a group and its implications have been studied extensively; see, for example, [5, 49].

In the paper [6], it was noted that the Transfer Current Theorem extends to the free and wired spanning forests if one uses the free and wired currents, respectively. To explain this, note that the orthocomplement of the row space $\star(G)$ of the vertex-edge incidence matrix of a finite graph $G$ is the kernel, denoted $\diamond(G)$, of the matrix. We call $\star(G)$ the star space of $G$ and $\diamond(G)$ the cycle space of $G$. For an infinite graph $G=(\mathrm{V}, \mathrm{E})$ exhausted by finite subgraphs $G_{n}$, we let $\star(G)$ be the closure of $\bigcup \star\left(G_{n}^{*}\right)$ and $\diamond(G)$ be the closure of $\bigcup \diamond\left(G_{n}\right)$, where we take the closure in $\ell^{2}(\mathrm{E})$. Then $\mathrm{WSF}_{G}$ is the determinantal probability measure corresponding to the projection $P_{\star(G)}$, while $\mathrm{FSF}_{G}$ is the determinantal probability measure

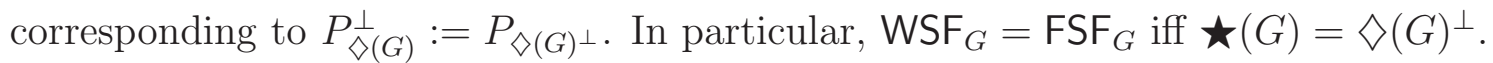

While the wired spanning forest is quite well understood, the free spanning forest measure is in general poorly understood. A more detailed summary of uniform spanning forest measures can be found in [36].

If $E$ is infinite and $Q_{n}$ are positive contractions on $\ell^{2}(E)$ that tend to $Q$ in the weak operator topology (i.e., in each matrix entry with respect to the standard orthonormal basis, we have convergence), then clearly $\mathbf{P}^{Q_{n}}$ tend to $\mathbf{P}^{Q}$ weak ${ }^{*}$. Since $\star\left(G_{n}^{*}\right) \subset \diamond\left(G_{n}\right)^{\perp}$, it follows from Theorem 2.1 that $\mathrm{WSF}_{G} \preccurlyeq \mathrm{FSF}_{G}$, though this was proved first by other means in $[6]$.

\section{Cayley graphs and Cayley diagrams}

For any group $\Gamma$ acting on a set $X$, if the contraction $Q$ on $\ell^{2}(X)$ is $\Gamma$-equivariant, then $\mathbf{P}^{Q}$ is $\Gamma$-invariant.

Definition 3.1. Let $\Gamma$ be a group. If $S$ is a set of elements of $\Gamma$, we write $S^{-1}:=$ $\left\{s^{-1} ; s \in S\right\}$. If $S=S^{-1}$ is such that the smallest subgroup of $\Gamma$ that contains all elements of $S$ is $\Gamma$ itself, then the corresponding Cayley graph of $\Gamma$ with respect to $S$ is the undirected graph whose vertex set is $\Gamma$ and whose edge set is $[\gamma, \gamma s]$ for $\gamma \in \Gamma$ and $s \in S$. The Cayley diagram $\operatorname{Cay}(\Gamma, S)$ contains more information and is a labelled oriented graph; namely, it is the graph whose vertex set is $\Gamma$ and whose edge set is $(\gamma, \gamma s)$ for $\gamma \in \Gamma$ and $s \in S$, where that edge is labelled s. Thus, for each unoriented edge of the Cayley graph, there are two oriented edges of the Cayley diagram, with inverse labels. We shall always assume that $S=S^{-1}$. An S-labelled graph is a graph each of whose edges is assigned a label in $S$.

A rooted graph $(G, o)$ is a graph $G$ with a distinguished vertex of $G$. We denote by $[(G, o)]$ the class of rooted graphs that are isomorphic to $G$ via an isomorphism that preserves 
the root. If $G$ is labelled or oriented, then we also require the isomorphism to preserve that extra structure. Generally we are interested in isomorphism classes and shall, after the first uses, drop the square brackets in the notation and thus not distinguish notationally between a rooted graph and its isomorphism class.

Given a vertex $v$ in a (possibly labelled and oriented) graph $G$ and $r \geq 0$, write $B(v, r ; G)$ for the (possibly labelled and oriented) graph induced by $G$ on the vertices within distance $r$ of $v$, with $v$ as the root. If $G$ is finite, let $\nu_{G, r}$ denote the law of $[B(v, r ; G)]$ when $v$ is chosen uniformly at random. If $G$ is a Cayley graph with identity element $o$ and $G_{n}$ are finite undirected graphs such that for every $r>0$, the laws $\nu_{G_{n}, r}$ tend to $\delta_{[B(o, r ; G)]}$, then we say that the random weak limit of $\left(G_{n}\right)_{n}$ is $G$.

We say that $\Gamma$ is sofic if there exists a sequence $\left(G_{n}\right)_{n \geq 1}$ of finite oriented graphs whose edges are labelled by elements of $S$ such that for every $r>0$, the laws $\nu_{G_{n}, r}$ tend to $\delta_{[B(o, r ; G)]}$, where $G$ is the Cayley diagram of $\Gamma$. In this case, we say that $\operatorname{Cay}(\Gamma, S)$ is a limit of finite $S$-labelled graphs. It is well known and easy to see that if $\operatorname{Cay}(\Gamma, S)$ is a limit of finite $S$-labelled graphs, then the same holds for any other finite generating set of elements $S^{\prime} \subset \Gamma$ $[59]$.

Definition 3.2. A S-labelled oriented graph $G$ is called an S-labelled Schreier graph if for each vertex in $G$ and each $s \in S$, there is precisely one incoming edge and one outgoing edge with the label s.

Let $\mathbf{F}_{S}$ denote the free group on the set $S$, i.e., $\mathbf{F}_{S}$ consists of formal products of letters $s^{ \pm}$with $s \in S$. We denote the neutral element of $\mathbf{F}_{S}$ by $\varnothing$. Each $S$-labelled Schreier graph is equipped with a natural action of $\mathbf{F}_{S}$. The action of $s \in S$ on $v \in \mathbf{V}$ yields the unique vertex $v^{\prime} \in \mathrm{V}$ such that there exists an $s$-labelled oriented edge $\left(v, v^{\prime}\right)$. We shall denote this vertex by v.s; and hence consider this action as a right action on $\mathrm{V}$. More generally, for $v \in \mathrm{V}$ and $w \in \mathbf{F}_{S}$, v.w denotes the vertex which is obtained by an oriented walk following the labels determined by $w \in \mathbf{F}_{S}$. Likewise, given an edge $e=(v, v . s)$ and $w \in \mathbf{F}_{S}$, write e.w $:=(v . w, v . w . s)$. For a set $A \subset \mathbf{F}_{S}$, write $v . A:=\{v . w ; w \in A\}$ and e.A $:=\{e . w ; w \in A\}$.

The following was proved in slightly different language by [19].

Lemma 3.3. Let $\Gamma$ be a group and $S$ a generating set of elements of $\Gamma$. The group $\Gamma$ is sofic if and only if $\operatorname{Cay}(\Gamma, S)$ is a limit of finite S-labelled Schreier graphs.

Suppose that $\theta$ is a probability measure on $\{0,1\}^{\mathrm{V}}$ or $\{0,1\}^{\mathrm{E}}$ for a graph $G=(\mathrm{V}, \mathrm{E})$, regarded as giving random subsets (of either vertices or edges) by using $\{0,1\}$-valued marks. For a vertex $v$ and $r \geq 0$, denote the restriction of $\theta$ to $B(v, r ; G)$ by $\theta(v, r)$. Write $[\theta(v, r)]$ for the probability measure induced on $[B(v, r ; G)]$. If $G$ is finite, let $\theta(G, r):=\frac{1}{|\mathrm{~V}|} \sum_{v \in \mathrm{V}}[\theta(v, r)]$. 
In other words, if $H$ is a rooted $\{0,1\}$-marked graph of radius at most $r$, then with $\cong$ denoting isomorphism of rooted marked graphs, $\theta(G, r)(H)=\frac{1}{|\mathrm{~V}|} \sum_{v \in \mathrm{V}} \sum_{H^{\prime} \cong H} \theta(v, r)\left(H^{\prime}\right)$. Suppose that $G=(\mathrm{V}, \mathrm{E})$ is the random weak limit of finite graphs $G_{n}=\left(\mathrm{V}_{n}, \mathrm{E}_{n}\right)$, that $\theta_{n}$ are probability measures on $\{0,1\}^{\mathrm{V}_{n}}$ or $\{0,1\}^{\mathrm{E}_{n}}$, and that $\theta$ is a probability measure on $\{0,1\}^{\mathrm{V}}$ or $\{0,1\}^{\mathrm{E}}$, respectively. If the weak* limit of $\theta_{n}\left(G_{n}, r\right)$ equals $\theta(o, r)$ for all $r \geq 0$, then we say that $\theta$ is the random weak limit of $\left(\theta_{n}\right)_{n}$.

Let $\Gamma$ be a group and $S$ be a finite generating set of elements of $\Gamma$. There is a natural action of $\Gamma$ on $\operatorname{Cay}(\Gamma, S)=(\Gamma, \mathrm{E})$ that we denote by $\lambda: \Gamma \rightarrow \operatorname{Aut}(\operatorname{Cay}(\Gamma, S))$. It is defined by the translations $\lambda(g)(h)=g h$ for $g, h \in \Gamma$. The vertex set $\Gamma$ of $\operatorname{Cay}(\Gamma, S)$ admits another $\Gamma$ action, which is given by the formula $\rho(g)(h)=h g^{-1}$ for $g, h \in \Gamma$. Both actions extend to unitary representations of $\Gamma$ on the Hilbert space $\ell^{2} \Gamma$, which we also denote by $\lambda$ and $\rho$. We denote the natural orthonormal basis of $\ell^{2} \Gamma$ by $\left\{\delta_{\gamma} ; \gamma \in \Gamma\right\}$. Since the multiplication in $\Gamma$ is associative, these actions commute. We define the (right) group von Neumann algebra of $\Gamma$ to be the algebra of $\Gamma$-equivariant operators,

$$
R(\Gamma):=\left\{T \in B\left(\ell^{2} \Gamma\right) ; \forall g \in \Gamma: \lambda(g) T=T \lambda(g)\right\},
$$

and note that $\rho(\Gamma) \subset R(\Gamma)$. We use $\rho$ also to denote the linearization $\rho: \mathbb{C} \Gamma \rightarrow R(\Gamma)$, which is a $*$-homomorphism, where $\mathbb{C} \Gamma$ denotes the complex group ring, which carries the natural involution that sends $g$ to $g^{-1}$ and is complex conjugation on the coefficients. The group von Neumann algebra $R(\Gamma)$ comes with a natural involution $T \mapsto T^{*}$ (the adjoint map) and a trace given by the formula

$$
\tau(T):=\left\langle T \delta_{o}, \delta_{o}\right\rangle,
$$

where $o$ is the identity element of $\Gamma$. The functional $\tau: R(\Gamma) \rightarrow \mathbb{C}$ leads to the definition of spectral measure: Let $T \in R(\Gamma)$ be a self-adjoint operator. There exists a unique probability measure $\mu_{T}$ on the interval $[-\|T\|,\|T\|] \subset \mathbb{R}$ such that

$$
\forall n \in \mathbb{N} \quad \tau\left(T^{n}\right)=\int t^{n} d \mu_{T}(t) .
$$

Note that the action of $\Gamma$ on $\mathrm{E}$ is isomorphic to the natural left action of $\Gamma$ on $\Gamma \times S$, where we identify a pair $(\gamma, s) \in \Gamma \times S$ with the edge $(\gamma, \gamma s) \in \mathrm{E}$. Abusing notation, we denote the natural unitary action of $\Gamma$ on $\ell^{2} E$ also by $\lambda$. We define the von Neumann algebra of the Cayley diagram $\operatorname{Cay}(\Gamma, S)$ to be

$$
R(\Gamma, S):=\left\{T \in B\left(\ell^{2} \mathrm{E}\right) ; \forall g \in \Gamma: \lambda(g) T=T \lambda(g)\right\} .
$$

LEMMA 3.4. There is a natural identification

$$
R(\Gamma, S)=M_{S}(R(\Gamma)),
$$

where $M_{S}(Z)$ denotes the $S \times S$-matrices over a ring $Z$. 
Proof. The isomorphism is described as follows. For $s \in S$, we denote the orthogonal projection onto $\ell^{2}(\{e \in \mathrm{E} ; e=(\gamma, \gamma s), \gamma \in \Gamma\})$ by $p_{s}$. Clearly, $p_{s}$ is $\lambda$-equivariant and there is a natural $\Gamma$-equivariant identification of $\{e \in \mathrm{E} ; e=(\gamma, \gamma s), \gamma \in \Gamma\}$ with $\Gamma$. Indeed, since $\operatorname{Cay}(\Gamma, S)$ is a Schreier graph, there is precisely one edge starting at $\gamma \in \Gamma$ with label $s \in S$. Let now $s, t \in S$. With a slight abuse of notation, every operator $T \in R(\Gamma, S)$ determines a matrix $\left(p_{s} T p_{t}\right)_{s, t \in S}$ of elements in $R(\Gamma)$. It is easily checked that this identification preserves all the structure.

Note that the von Neumann algebra $R(\Gamma, S)$ also comes equipped with a natural trace $\tau_{S}: R(\Gamma, S) \rightarrow \mathbb{C}$ given by $\tau_{S}(T):=\sum_{s \in S} \tau\left(p_{s} T p_{s}\right)$ with $p_{s}$ being as defined in the proof of Lemma 3.4; we call $\tau_{S}$ the natural extension of $\tau$.

Let now $G=(\mathrm{V}, \mathrm{E})$ be an $S$-labelled Schreier graph. We consider the algebra $B\left(\ell^{2} \mathrm{~V}\right)$ of bounded operators on the Hilbert space $\ell^{2} \mathrm{~V}$ and note that we have a natural identification $B\left(\ell^{2} \mathrm{E}\right)=M_{S}\left(B\left(\ell^{2} \mathbf{V}\right)\right)$ as in the proof of the previous lemma. If $\mathbf{V}$ is finite, then $B\left(\ell^{2} \mathbf{V}\right)$ carries a natural normalized trace

$$
\operatorname{tr}_{\mathrm{V}}(T):=\frac{1}{|\mathrm{~V}|} \sum_{v \in \mathrm{V}}\left\langle T \delta_{v}, \delta_{v}\right\rangle .
$$

Similarly, there is then a natural $\operatorname{trace}^{\operatorname{tr}_{\mathrm{E}}}$ on the algebra $B\left(\ell^{2} \mathrm{E}\right)$ when $\mathrm{E}$ is finite. Every element $w \in \mathbf{F}_{S}$ determines a unitary operator $\pi(w)$ on $\ell^{2}(\mathrm{~V})$ for each $S$-labelled Schreier graph $G=(\mathrm{V}, \mathrm{E})$. It is given by linearity and the formula

$$
\forall w \in \mathbf{F}_{S} \quad \forall v \in \mathrm{V} \quad \pi(w)\left(\delta_{v}\right):=\delta_{v \cdot w^{-1}} .
$$

By taking linear combinations, every element in the complex group ring $a \in \mathbb{C F}_{S}$ determines a bounded operator $\rho_{G}(a)$ in $B\left(\ell^{2} \bigvee\right)$. Note that if $G=\operatorname{Cay}(\Gamma, S)$, then $\rho_{G}(a)=\rho(\pi(a))$, where $\pi: \mathbb{C F}_{S} \rightarrow \mathbb{C} \Gamma$ denotes the natural homomorphism of group rings extending the map from $S$ to its image in $\Gamma$.

The following lemma is well known [20]; let us include a proof for convenience.

Lemma 3.5. Let $\Gamma$ be a sofic group and $S$ be a finite generating set of elements in $\Gamma$. Let $\left(G_{n}\right)_{n}$ be a sequence of finite $S$-labelled Schreier graphs whose limit is $G:=\operatorname{Cay}(\Gamma, S)$. Then for each $a \in \mathbb{C F}_{S}$, we have

$$
\tau\left(\rho_{G}(a)\right)=\lim _{n \rightarrow \infty} \operatorname{tr}_{\vee_{n}}\left(\rho_{G_{n}}(a)\right) .
$$

Proof. It is enough to prove the statement for $a=w \in \mathbf{F}_{S}$. Then the left-hand side is 1 or 0 depending on whether $\pi(w)=o$ in $\Gamma$ or not. The right-hand side equals

$$
\lim _{n \rightarrow \infty} \frac{1}{\left|\mathrm{~V}_{n}\right|} \sum_{v \in \mathrm{V}_{n}}\left\langle\rho_{G_{n}}(w) \delta_{v}, \delta_{v}\right\rangle
$$


and thus measures the fraction of vertices that are fixed by the action of $\rho_{G_{n}}(w)$. If $w^{-1}=$ $s_{1}^{\varepsilon_{1}} \cdots s_{n}^{\varepsilon_{n}}$ with $s_{1}, \ldots, s_{n} \in S$ and $\varepsilon_{1}, \ldots, \varepsilon_{n} \in\{ \pm 1\}$, then this is the fraction of vertices $v \in \mathrm{V}_{n}$ for which an oriented walk with labels $s_{1}^{\varepsilon_{1}}, \ldots, s_{n}^{\varepsilon_{n}}$ ends at $v \in \mathrm{V}_{n}$, i.e., if $v \cdot w^{-1}=v$ or not. It is clear that this fraction converges to 1 or 0 depending on whether the corresponding walk in $G$ starting at $o \in \Gamma$ ends at $o \in \Gamma$ or not. It ends at $o$ if and only if $\pi(w)=o$ in $\Gamma$. Hence, the left- and the right-hand sides are equal. This finishes the proof.

\section{Tracial von Neumann algebras and their ultraproducts}

Before we introduce the metric ultraproduct of tracial von Neumann algebras, we shall recall some basic facts about von Neumann algebras. A von Neumann algebra is defined to be a weakly closed unital $*$-subalgebra of the space $B(H)$ of bounded linear operators on some Hilbert space $H$. Note that the weak topology here refers to the weak operator topology (WOT).

If $K \subset B(H)$ is a self-adjoint subset, then we define the commutant of $K$ as $K^{\prime}:=\{T \in$ $B(H) ; \forall S \in K S T=T S\}$. It is easy to see that $K^{\prime}$ is a von Neumann algebra. Most von Neumann algebras arise naturally as the commutant of some explicit set of operators. For example, we defined $R(\Gamma)=\left\{\lambda(\gamma) \in B\left(\ell^{2} \Gamma\right) ; \gamma \in \Gamma\right\}^{\prime}$. Von Neumann's Double Commutant Theorem says that this construction exhausts all von Neumann algebras; more specifically, $M \subset B(H)$ is a von Neumann algebra if and only if $M=M^{*}$ and $M=M^{\prime \prime}$.

Since any weakly closed algebra is also norm closed in $B(H)$, a von Neumann algebra is in particular a $C^{*}$-algebra. Like $C^{*}$-algebras, von Neumann algebras admit an abstract characterization. A $C^{*}$-algebra $M$ is $*$-isomorphic to a von Neumann algebra if and only if as a Banach space, $M$ is a dual Banach space; in this case, the pre-dual $M_{*}$ is unique up to isometry. In case $M=B(H)$, the pre-dual $M_{*}$ is the Banach space of trace-class operators on $H$ with the natural pairing between $M$ and $M_{*}$ given by the trace. It is well known that the weak* topology (seeing $B(H)$ as a dual Banach space) is nothing but the so-called ultra-weak topology. Moreover, if $M$ is a weakly closed sub-algebra of $B(H)$, then the natural weak* topology on $M=\left(M_{*}\right)^{*}$ can be identified with the ultra-weak topology inherited from $B(H)$.

Following Dedekind's characterization (or rather definition) of an infinite set, a projection (which always means "orthogonal projection" for us) $P \in M$ in a von Neumann algebra is called infinite if it is equivalent in $M$ to a subprojection of itself, i.e., if there exists a partial isometry $U \in M$ such that $U^{*} U=P$ and $U U^{*}$ is a proper subprojection of $P$. A projection that is not infinite is called finite. For our purposes, only finite von Neumann algebras matter, and these are (by definition) the ones in which every projection is finite. Note that $B\left(\ell^{2} \mathbf{V}\right)$ is finite if and only if $\mathbf{V}$ is finite. 
A certificate for finiteness of a von Neumann algebra $M$ is the existence of a positive, faithful trace $\tau: M \rightarrow \mathbb{C}$. Here, a (linear) functional $\tau: M \rightarrow \mathbb{C}$ is called positive if $\tau\left(T^{*} T\right) \geq 0$. A positive functional is called faithful if $\tau\left(T^{*} T\right)=0$ implies $T=0$. The functional $\tau$ is called a trace if $\tau(T S)=\tau(S T)$ for all $S, T \in M$. For the sake of getting used to the definitions, let us convince ourselves that a von Neumann algebra $M$ with a positive and faithful trace $\tau: M \rightarrow \mathbb{C}$ is finite. Let $P$ be a projection and $U$ be a partial isometry with $U^{*} U=P$. Suppose that $U U^{*}$ is a subprojection of $P$. Then $Q:=P-U U^{*}$ is also a projection and hence $Q=Q^{2}=Q^{*} Q$. However, $\tau\left(Q^{*} Q\right)=\tau(Q)=\tau\left(U^{*} U-U U^{*}\right)=0$ and hence $Q=0$. This shows that $U U^{*}=P$. Hence, $M$ is a finite von Neumann algebra.

A functional $\tau: M \rightarrow \mathbb{C}$ is called normal if it is ultra-weakly continuous; natural examples are sums of so-called vector states $\tau(T):=\sum_{i}\left\langle T \xi_{i}, \xi_{i}\right\rangle$ for $\xi_{1}, \ldots, \xi_{n} \in H$. The functional is called unital if $\tau(1)=1$. We call a pair $(M, \tau)$ a tracial von Neumann algebra if $M$ is a von Neumann algebra and $\tau: M \rightarrow \mathbb{C}$ is a normal, positive, faithful, and unital trace. One can show that $\tau: R(\Gamma) \rightarrow \mathbb{C}$ is a normal, positive, faithful, and unital trace. Hence, $R(\Gamma)$ is a finite von Neumann algebra.

A $*$-homomorphism $\varphi:\left(N, \tau_{N}\right) \rightarrow\left(M, \tau_{M}\right)$ between $*$-algebras equipped with traces is called trace preserving if $\tau_{N}=\tau_{M} \circ \varphi$. If $\tau: M \rightarrow \mathbb{C}$ is a normal, positive, faithful, and unital trace, then the norm $T \mapsto \tau\left(T^{*} T\right)^{1 / 2}$ determines the ultra-weak topology on norm-bounded sets. In particular, this implies that if $\left(N, \tau_{N}\right)$ and $\left(M, \tau_{M}\right)$ are tracial von Neumann algebras, $N_{0} \subset\left(N, \tau_{N}\right)$ is a dense $*$-subalgebra, and $\tilde{\varphi}:\left(N_{0}, \tau_{N}\left\lceil N_{0}\right) \rightarrow\left(M, \tau_{M}\right)\right.$ is a trace-preserving $*$-homomorphism, then $\tilde{\varphi}$ has a unique extension to a trace-preserving *-homomorphism $\varphi:\left(N, \tau_{N}\right) \rightarrow\left(M, \tau_{M}\right)$. For all these facts about von Neumann algebras and more, see [56] and the references therein.

The following construction of metric ultraproducts of von Neumann algebras preserves the class of tracial von Neumann algebras and plays a crucial role in their study.

Definition 4.1. Let $\left(M_{n}, \tau_{n}\right)$ be a sequence of tracial von Neumann algebras. Let $\omega$ be a non-principal ultrafilter on $\mathbb{N}$. We consider

$$
\ell^{\infty}\left(\mathbb{N},\left(M_{n}, \tau_{n}\right)_{n}\right):=\left\{\left(T_{n}\right)_{n} ; T_{n} \in M_{n}, \sup \left\{\left\|T_{n}\right\| ; n \in \mathbb{N}\right\}<\infty\right\}
$$

and set

$$
J_{\omega}:=\left\{\left(T_{n}\right)_{n} \in \ell^{\infty}\left(\mathbb{N},\left(M_{n}, \tau_{n}\right)_{n}\right) ; \lim _{n \rightarrow \omega} \tau_{n}\left(T_{n}^{*} T_{n}\right)=0\right\} .
$$

It is well known and easy to verify that $J_{\omega} \subset \ell^{\infty}\left(\mathbb{N},\left(M_{n}, \tau_{n}\right)_{n}\right)$ is a two-sided $*$-ideal. The metric ultraproduct of $\left(M_{n}, \tau_{n}\right)$ is defined to be the quotient

$$
\prod_{n \rightarrow \omega}\left(M_{n}, \tau_{n}\right):=\frac{\ell^{\infty}\left(\mathbb{N},\left(M_{n}, \tau_{n}\right)_{n}\right)}{J_{\omega}} \quad \text { with trace } \tau_{\omega}\left(\left(T_{n}\right)_{n}+J_{\omega}\right):=\lim _{n \rightarrow \omega} \tau_{n}\left(T_{n}\right) .
$$


It has been shown by Connes [15, Section I.3] that $\left(\prod_{n \rightarrow \omega}\left(M_{n}, \tau_{n}\right), \tau_{\omega}\right)$ is again a von Neumann algebra acting on a concrete Hilbert space ${ }_{\omega} H$. This is not straightforward, sinceif $M_{n} \subset B\left(H_{n}\right)$ - the ultraproduct $\prod_{n \rightarrow \omega}\left(M_{n}, \tau_{n}\right)$ does not act on the Hilbert space arising as the ordinary Banach space ultraproduct $H_{\omega}:=\prod_{n \rightarrow \omega} H_{n}$ of the sequence of Hilbert spaces $\left(H_{n}\right)_{n}$. One can show that $\tau_{\omega}: \prod_{n \rightarrow \omega}\left(M_{n}, \tau_{n}\right) \rightarrow \mathbb{C}$ is a normal, positive, faithful, and unital trace.

In the context of the previous definition, we shall say that a sequence $\left(T_{n}\right)_{n}$ of operators with $T_{n} \in M_{n}$ represents some operator $T$ in the ultraproduct if $\left(T_{n}\right)_{n} \in \ell^{\infty}\left(\mathbb{N},\left(M_{n}, \tau_{n}\right)_{n}\right)$ and $T$ is its residue class modulo $J_{\omega}$. Since $J_{\omega}$ is a two-sided $*$-ideal, we get: If $\left(T_{n}\right)_{n}$ represents $T$ and $\left(S_{n}\right)_{n}$ represents $S$, then $\left(T_{n}^{*}\right)_{n}$ represents $T^{*},\left(T_{n}+S_{n}\right)_{n}$ represents $T+S$, and $\left(T_{n} S_{n}\right)_{n}$ represents $T S$. The following lemma is only slightly more involved. Recall that if $T$ is a self-adjoint operator and $f: \mathbb{R} \rightarrow \mathbb{R}$ is continuous, then $f(T)$ is defined by using the spectral decomposition of $T$.

LEMMA 4.2. Let $\left(T_{n}\right)_{n}$ be a sequence of self-adjoint operators in $\ell^{\infty}\left(\mathbb{N},\left(M_{n}, \tau_{n}\right)_{n}\right)$ representing some operator $T$ in the ultraproduct. Let $f: \mathbb{R} \rightarrow \mathbb{R}$ be a continuous function. Then the sequence $\left(f\left(T_{n}\right)\right)_{n}$ represents $f(T)$. Let $\left(S_{n}\right)_{n}$ be another sequence in $\ell^{\infty}\left(\mathbb{N},\left(M_{n}, \tau_{n}\right)_{n}\right)$ of self-adjoint operators representing some operator $S$ in the ultraproduct. If $S_{n} \leq T_{n}$ for all $n \in \mathbb{N}$, then $S \leq T$.

Proof. By the above, the first statement is clear for polynomial functions. It follows for general continuous functions by the Stone-Weierstrass approximation theorem. Note that $S_{n} \leq T_{n}$ if and only if $T_{n}-S_{n}=C_{n}^{*} C_{n}$ for some $C_{n} \in M_{n}$. Since $\left(S_{n}\right)_{n},\left(T_{n}\right)_{n} \in$ $\ell^{\infty}\left(\mathbb{N},\left(M_{n}, \tau_{n}\right)_{n}\right)$, we also have $\left(C_{n}\right)_{n} \in \ell^{\infty}\left(\mathbb{N},\left(M_{n}, \tau_{n}\right)_{n}\right)$. Let $C$ be the operator that is represented by the sequence $\left(C_{n}\right)_{n}$. Then $T-S=C^{*} C$ and hence $S \leq T$. This finishes the proof.

REMARK 4.3. The definition of metric ultraproduct is made in such a way that it is compatible with spectral measures in the following sense. If $\left(T_{n}\right)_{n}$ is a sequence of selfadjoint operators in $\ell^{\infty}\left(\mathbb{N},\left(M_{n}, \tau_{n}\right)_{n}\right)$ that represents some self-adjoint operator $T$ in the ultraproduct, then by definition and the above remarks, $\lim _{n \rightarrow \omega} \tau_{n}\left(T_{n}^{k}\right)=\tau_{\omega}\left(T^{k}\right)$ for all $k \geq 0$, whence $\lim _{n \rightarrow \omega} \mu_{T_{n}}=\mu_{T}$ in the weak ${ }^{*}$ topology.

The following proposition summarizes and extends results from [20].

Proposition 4.4. Let $\Gamma$ be a sofic group and $S$ be a finite generating set of elements in $\Gamma$. Let $\left(G_{n}\right)_{n}$ be a sequence of finite S-labelled Schreier graphs whose limit is $G:=\operatorname{Cay}(\Gamma, S)$. There exist trace-preserving embeddings

$$
\iota:(R(\Gamma), \tau) \rightarrow \prod_{n \rightarrow \omega}\left(B\left(\ell^{2} \mathrm{~V}_{n}\right), \operatorname{tr}_{\mathrm{V}_{n}}\right) \quad \text { and } \quad \iota_{S}:\left(R(\Gamma, S), \tau_{S}\right) \rightarrow \prod_{n \rightarrow \omega}\left(B\left(\ell^{2} \mathrm{E}_{n}\right), \operatorname{tr}_{\mathrm{E}_{n}}\right)
$$


Moreover, if $\left(T_{n}\right)_{n} \in \ell^{\infty}\left(\mathbb{N},\left(B\left(\ell^{2} \bigvee_{n}\right), \operatorname{tr}_{\vee_{n}}\right)\right)$ represents $\iota(T)$ for some $T \in R(\Gamma)$, then

$$
\lim _{n \rightarrow \omega}\left|\mathrm{V}_{n}\right|^{-1} \sum_{v \in \mathrm{V}_{n}}\left|\left\langle T_{n} \delta_{v \cdot \gamma}, \delta_{v \cdot \gamma^{\prime}}\right\rangle-\left\langle T \delta_{o \cdot \gamma}, \delta_{o \cdot \gamma^{\prime}}\right\rangle\right|=0
$$

for all $\gamma, \gamma^{\prime} \in \mathbf{F}_{S}$. Similarly, if $\left(T_{n}\right)_{n} \in \ell^{\infty}\left(\mathbb{N},\left(B\left(\ell^{2} \mathrm{E}_{n}\right), \operatorname{tr}_{\mathrm{E}_{n}}\right)\right)$ represents $\iota_{S}(T)$ for some $T \in R(\Gamma, S)$, then

$$
\lim _{n \rightarrow \omega}\left|\mathrm{V}_{n}\right|^{-1} \sum_{v \in \mathrm{V}_{n}}\left|\left\langle T_{n} \delta_{(v \cdot \gamma, v \cdot \gamma s)}, \delta_{\left(v \cdot \gamma^{\prime}, v \cdot \gamma^{\prime} s^{\prime}\right)}\right\rangle-\left\langle T \delta_{(o \cdot \gamma, o . \gamma s)}, \delta_{\left(o \cdot \gamma^{\prime}, o \cdot \gamma^{\prime} s^{\prime}\right)}\right\rangle\right|=0
$$

for all $\gamma, \gamma^{\prime} \in \mathbf{F}_{S}$ and $s, s^{\prime} \in S$.

Embedding theorems like the preceding proposition were first proved by Elek and Szabó; see, for example, [20, Theorem 2]. However, our emphasis is on the special features of embeddings coming from a sofic approximation. In particular, we are interested in Equations (3) and (4), which are not just features of every trace-preserving embedding.

Proof of Proposition 4.4: For each $n \in \mathbb{N}$, we consider the $*$-homomorphism

$$
\rho_{G_{n}}: \mathbb{C F}_{S} \rightarrow\left(B\left(\ell^{2} \mathrm{~V}_{n}\right), \operatorname{tr}_{\mathrm{V}_{\mathrm{n}}}\right)
$$

This sequence induces a $*$-homomorphism

$$
\rho_{\omega}: \mathbb{C F}_{S} \rightarrow \prod_{n \rightarrow \omega}\left(B\left(\ell^{2} \mathrm{~V}_{n}\right), \operatorname{tr}_{\vee_{n}}\right)
$$

We denote the canonical trace on $\prod_{n \rightarrow \omega}\left(B\left(\ell^{2} \bigvee_{n}\right), \operatorname{tr}_{\vee_{n}}\right)$ by $\operatorname{tr}_{\omega}:=\lim _{n \rightarrow \omega} \operatorname{tr}_{\mathrm{V}_{n}}$. Since $\left(G_{n}\right)_{n}$ is a sofic approximation to $\Gamma$, we obtain

$$
\tau_{\omega}\left(\rho_{\omega}(w)\right)= \begin{cases}1 & \text { if } \pi(w)=e \text { in } \Gamma \\ 0 & \text { if } \pi(w) \neq e \text { in } \Gamma .\end{cases}
$$

As the result depends only on $\pi(w)$, this shows that $\rho_{\omega}$ descends to a $*$-homomorphism $\rho_{\omega}: \mathbb{C} \Gamma \rightarrow \prod_{n \rightarrow \omega}\left(B\left(\ell^{2} \vee_{n}\right), \operatorname{tr}_{\vee_{n}}\right)$ that preserves the canonical trace on $\mathbb{C} \Gamma$. Note also that $\rho(\mathbb{C} \Gamma) \subset R(\Gamma)$ is weakly dense. Indeed, this is a standard fact and follows, for example, from the Commutation Theorem [17, Theorem 1 on page 80]. If there is no risk of confusion, we shall sometimes identify $\mathbb{C} \Gamma$ with its image in $R(\Gamma)$. It is a standard fact-see the remarks before Definition 4.1 - that $\rho_{\omega}$ has an extension $\iota$ to the group von Neumann $R(\Gamma)$ of $\Gamma$.

The second embedding is obtained after passing to matrix algebras $M_{S}(\bullet)$ via the natural isomorphisms $M_{S} R(\Gamma)=R(\Gamma, S)$ and $M_{S}\left(B\left(\ell^{2} \bigvee_{n}\right)\right)=B\left(\ell^{2} \mathrm{E}\right)$.

We shall now prove Equation (3). Upon replacing $T$ by $\rho_{G}\left(\gamma^{\prime}\right) T \rho_{G}\left(\gamma^{-1}\right)$ and $T_{n}$ by $\rho_{G_{n}}\left(\gamma^{\prime}\right) T_{n} \rho_{G_{n}}\left(\gamma^{-1}\right)$, we may assume that $\gamma=\gamma^{\prime}=o$. We first study the case $T=0$. We 
need to show that

$$
\lim _{n \rightarrow \omega}\left|\mathrm{V}_{n}\right|^{-1} \sum_{v \in \mathrm{V}_{n}}\left|\left\langle T_{n} \delta_{v}, \delta_{v}\right\rangle\right|=0
$$

We may write $T_{n}:=\sum_{k=0}^{3} i^{k} T_{n}^{(k)}$ with $T_{n}^{(k)}$ positive for each $0 \leq k \leq 3$ and $n \in \mathbb{N}$. Here, e.g., $T_{n}^{(0)}-T_{n}^{(2)}=\Re\left(T_{n}\right):=\left(T_{n}+T_{n}^{*}\right) / 2$ are self-adjoint operators that form a sequence representing $\left(T+T^{*}\right) / 2=0$, and $T_{n}^{(0)}=\left(\Re T_{n}\right)_{+}$is a function of $\Re T_{n}$. By Lemma 4.2 and the remarks preceding it, each sequence $\left(T_{n}^{(k)}\right)_{n}$ represents zero in the ultraproduct. Hence, Equation (5) holds for $\left(T_{n}^{(k)}\right)_{n}$ for $0 \leq k \leq 3$ because all scalar products are positive already, and hence $(5)$ holds for $\left(T_{n}\right)_{n}$ by the triangle inequality.

We conclude again from the triangle inequality that if Equation (3) holds for one sequence $\left(T_{n}\right)_{n}$ representing some operator $\iota(T)$ in the ultraproduct, then it holds for every sequence representing $\iota(T)$. Thus, whether or not Equation (3) holds is a property of $\iota(T) \in \prod_{n \rightarrow \omega}\left(B\left(\ell^{2} \bigvee_{n}\right), \operatorname{tr}_{\vee_{n}}\right)$ alone.

It is clear that Equation (3) holds for $\iota(\rho(\gamma))$ for $\gamma \in \Gamma$, as follows directly from the fact that the limit of $\left(G_{n}\right)_{n}$ is $G$. It is also clear that the set of operators $T \in R(\Gamma)$ for which Equation (3) holds is closed under addition and multiplication by scalars. Thus, Equation (3) holds for the complex group ring $\mathbb{C} \Gamma \subset R(\Gamma)$ and every ultrafilter. Moreover, our construction shows that for each $T \in \mathbb{C} \Gamma$, there exists a sequence $\left(T_{n}\right)_{n} \in \ell^{\infty}\left(\mathbb{N},\left(B\left(\ell^{2} \bigvee_{n}\right), \operatorname{tr}_{\mathrm{V}_{\mathrm{n}}}\right)\right)$ such that

$$
\lim _{n \rightarrow \infty}\left|\mathrm{V}_{n}\right|^{-1} \sum_{v \in \mathrm{V}_{n}}\left|\left\langle T_{n} \delta_{v}, \delta_{v}\right\rangle-\left\langle T \delta_{o}, \delta_{o}\right\rangle\right|=0
$$

Moreover, $\left(T_{n}\right)_{n}$ represents $\iota(T)$ in the ultraproduct with respect to any ultrafilter. Now, let $T \in R(\Gamma)$ and let us choose a sequence $\left(S_{i}\right)_{i}$ with $S_{i} \in \mathbb{C} \Gamma, S_{i} \rightarrow T$ in the weak operator topology and $\sup _{i}\left\|S_{i}\right\| \leq\|T\|$. For each $i$, let $\left(S_{i, n}\right)_{n}$ be a sequence that represents $\iota\left(S_{i}\right)$ in the ultraproduct. It is now clear that if the sequence $\left(k_{n}\right)_{n}$ of natural numbers increases slowly enough, then $\left(S_{k_{n}, n}\right)_{n}$ represents $\iota(T)$ in the ultraproduct and that Equation (3) holds for this sequence. Since we have shown that Equation (3) depends only on $\iota(T)$, it follows that Equation (3) holds for any sequence $\left(T_{n}\right)_{n}$ that represents $\iota(T)$ in the ultraproduct. This proves Equation (3) for every $T \in R(\Gamma)$. The proof of the second equality is similar. This finishes the proof of the proposition.

The following lemma shows that our previous result is useful to connect the approximation of some operator $\iota(T)$ by a sequence $\left(T_{n}\right)_{n}$ to an approximation of the associated determinantal probability measures.

Lemma 4.5. Let $\Gamma$ be a sofic group and $S$ be a finite generating set of elements in $\Gamma$. Let $\left(G_{n}\right)_{n}$ be a sequence of finite S-labelled Schreier graphs whose limit is $G:=\operatorname{Cay}(\Gamma, S)$. Let $\iota$ and $\iota_{S}$ be trace-preserving embeddings as in Proposition 4.4. Let $T \in R \Gamma$ be such that 
$0 \leq T \leq I$ and suppose that $\left(T_{n}\right)_{n}$ represents $\iota(T)$ in the ultraproduct $\prod_{n \rightarrow \omega}\left(B\left(\ell^{2} \mathrm{~V}_{n}\right), \operatorname{tr}_{\mathrm{V}_{n}}\right)$ with $0 \leq T_{n} \leq I$ for each $n \in \mathbb{N}$. Then $\lim _{n \rightarrow \omega} \mathbf{P}^{T_{n}}=\mathbf{P}^{T}$ in the random weak topology, in other words,

$$
\lim _{n \rightarrow \omega} \frac{1}{\left|\mathrm{~V}_{n}\right|} \sum_{v \in \mathrm{V}_{n}} \mathbf{P}^{T_{n}}[v \cdot A \subset \mathfrak{S}]=\mathbf{P}^{T}[o . A \subset \mathfrak{S}]
$$

for all finite $A \subset \mathbf{F}_{S}$. Likewise, if $T \in R(\Gamma, S)$ is such that $0 \leq T \leq I$ and $\left(T_{n}\right)_{n}$ represents $\iota_{S}(T)$ in the ultraproduct $\prod_{n \rightarrow \omega}\left(B\left(\ell^{2} \mathrm{E}_{n}\right), \operatorname{tr}_{\mathrm{E}_{n}}\right)$ with $0 \leq T_{n} \leq I$ for each $n \in \mathbb{N}$, then $\lim _{n \rightarrow \omega} \mathbf{P}^{T_{n}}=\mathbf{P}^{T}$ in the random weak topology; in other words, given a finite set $A_{s} \subset \mathbf{F}_{S}$ for each $s \in S$, we have

$$
\lim _{n \rightarrow \omega} \frac{1}{\left|\mathrm{~V}_{n}\right|} \sum_{v \in \mathrm{V}_{n}} \mathbf{P}^{T_{n}}\left[\bigcup_{s \in S}(v, v \cdot s) \cdot A_{s} \subset \mathfrak{S}\right]=\mathbf{P}^{T}\left[\bigcup_{s \in S}(o, s) \cdot A_{s} \subset \mathfrak{S}\right] .
$$

Proof. We prove the first statement, as the second is similar and just involves more notation.

By definition of determinantal probability measures, the desired identity is the same as

$$
\lim _{n \rightarrow \omega} \frac{1}{\left|\mathrm{~V}_{n}\right|} \sum_{v \in \mathrm{V}_{n}} \operatorname{det}\left(T_{n}\lceil\text { v.A })=\operatorname{det}(T\lceil o . A) .\right.
$$

Because $G$ is the limit of $\left(G_{n}\right)_{n}$, we may assume that $|o . A|=|A|$, i.e., that $\pi$ is injective on A. Define

$$
a_{n}\left(v, \gamma, \gamma^{\prime}\right):=\left|\left\langle T_{n} \delta_{v \cdot \gamma}, \delta_{v \cdot \gamma^{\prime}}\right\rangle-\left\langle T \delta_{\gamma}, \delta_{\gamma^{\prime}}\right\rangle\right|
$$

By Equation (3), we have

$$
\lim _{n \rightarrow \omega} \frac{1}{\left|\mathrm{~V}_{n}\right|} \sum_{v \in \mathrm{V}_{n}} \sum_{\gamma, \gamma^{\prime} \in A} a_{n}\left(v, \gamma, \gamma^{\prime}\right)=0
$$

Since $a_{n}\left(v, \gamma, \gamma^{\prime}\right) \leq 2$, it follows that

$$
\lim _{n \rightarrow \omega} \frac{1}{\left|\mathrm{~V}_{n}\right|} \sum_{v \in \mathrm{V}_{n}} \prod_{\gamma^{\prime} \in A} \sum_{\gamma \in A} a_{n}\left(v, \gamma, \gamma^{\prime}\right)=0,
$$

whence by Hadamard's inequality,

$$
\lim _{n \rightarrow \omega} \frac{1}{\left|\mathrm{~V}_{n}\right|} \sum_{v \in \mathrm{V}_{n}} \mid \operatorname{det}\left(T_{n}\lceil v \cdot A)-\operatorname{det}(T\lceil o . A) \mid=0,\right.
$$

which is stronger than the limit we desired.

The following lemma is well known [18], but we provide a proof for the convenience of the reader, as it is an essential tool for our proofs.

Lemma 4.6. Let $H$ be a Hilbert space, $M \subseteq B(H)$ be a von Neumann algebra, and $S, T \in M$ with $0 \leq S \leq T \leq I$. Then there exists a positive contraction $C \in M$ with $T^{1 / 2} C T^{1 / 2}=S$. 
Proof. Let $H_{0} \subset H$ be the closure of the image of $T$ and note that $H_{0}=\operatorname{ker}(T)^{\perp}$. Since the orthogonal projection onto $H_{0}$ is contained in $M$, we may assume without loss of generality that $H_{0}=H$. The space $H_{0}^{\prime}:=\left\{T^{1 / 2} \xi ; \xi \in H\right\}$ is dense in $H_{0}$. We define $D$ to be $D\left(T^{1 / 2} \xi\right):=S^{1 / 2} \xi$ on $H_{0}^{\prime}$. Now, since $0 \leq S \leq T$, it is easy to see that $D$ is well defined and extends to a contraction. Indeed,

$$
\left\|D\left(T^{1 / 2} \xi\right)\right\|^{2}=\left\langle D\left(T^{1 / 2} \xi\right), D\left(T^{1 / 2} \xi\right)\right\rangle=\left\langle S^{1 / 2} \xi, S^{1 / 2} \xi\right\rangle=\langle S \xi, \xi\rangle \leq\langle T \xi, \xi\rangle=\left\|T^{1 / 2} \xi\right\|^{2} .
$$

The inequality shows that $D$ is well defined; the whole shows that $D$ is contractive on $H_{0}^{\prime}$ and hence has a unique contractive extension to $H$. It is also clear that $D T^{1 / 2}=S^{1 / 2}$ on $H$. For every operator $U \in M^{\prime}$, we get

$$
U D T^{1 / 2}=U S^{1 / 2}=S^{1 / 2} U=D T^{1 / 2} U=D U T^{1 / 2} .
$$

Hence, $U D=D U$ on the image of $T^{1 / 2}$, which we assume is dense in $H$. This implies $D \in M^{\prime \prime}$ and hence $D \in M$, since $M$ is a von Neumann algebra. We can now set $C:=D^{*} D \geq 0$, note that $C \in M$, and that we have $T^{1 / 2} C T^{1 / 2}=T^{1 / 2} D^{*} D T^{1 / 2}=S^{1 / 2} S^{1 / 2}=S$. It is obvious that $C$ is also a contraction. This finishes the proof of the lemma.

The previous lemma can now be used to show that self-adjoint operators $S, T$ in the ultra-product with $0 \leq S \leq T \leq I$ can be approximated by sequences satisfying the same relation.

Lemma 4.7. Let $\left(M_{n}, \tau_{n}\right)$ be a sequence of tracial von Neumann algebras. Let $S, T \in$ $\prod_{n \rightarrow \omega}\left(M_{n}, \tau_{n}\right)$ be operators such that $0 \leq S \leq T \leq I$. Then there exist sequences $\left(T_{n}\right)_{n}$ and $\left(S_{n}\right)_{n}$ with $T_{n}, S_{n} \in M_{n}$ that represent $T$ and $S$ in the ultraproduct and such that $0 \leq S_{n} \leq$ $T_{n} \leq I$ for each $n \in \mathbb{N}$.

Proof. First of all, by Lemma 4.6 there exists a positive contraction $C \in \prod_{n \rightarrow \omega}\left(M_{n}, \tau_{n}\right)$ such that $T^{1 / 2} C T^{1 / 2}=S$. Let $\left(T_{n}\right)_{n}$ be some representative of $T$. By Lemma $4.2,\left(\left(T_{n}^{*} T_{n}\right)^{1 / 2}\right)_{n}$ represents $\left(T^{*} T\right)^{1 / 2}=T$, so by replacing $T_{n}$ with $\left(T_{n}^{*} T_{n}\right)^{1 / 2}$, we may assume that $T_{n} \geq 0$. Let $\epsilon_{n}:=\mu_{T_{n}}((1, \infty))$, where $\mu_{T_{n}}$ denotes the spectral measure of $T_{n}$. Since $T \leq I$, we know that $\epsilon_{n} \rightarrow 0$ as $n \rightarrow \infty$. Let $Q_{n}$ be the spectral projection onto the interval [0,1]; thus, $\tau_{n}\left(Q_{n}\right)=1-\epsilon_{n} \rightarrow 1$ as $n \rightarrow \infty$. This implies that $\left(Q_{n}\right)_{n}$ represents $I$ in the ultraproduct. Hence, we may replace $T_{n}$ by $Q_{n} T_{n}$ to obtain a sequence $\left(T_{n}\right)_{n}$ that represents $T$ and satisfies $0 \leq T_{n} \leq I$ for all $n \in \mathbb{N}$. The same argument applies to $0 \leq C \leq I$ and we obtain a sequence $\left(C_{n}\right)_{n}$ representing $C$ such that $0 \leq C_{n} \leq I$ for all $n \in \mathbb{N}$. Moreover, the sequence $\left(S_{n}\right)_{n}$ with $S_{n}:=T_{n}^{1 / 2} C_{n} T_{n}^{1 / 2}$ represents $S=T^{1 / 2} C T^{1 / 2}$. Now $0 \leq C_{n} \leq I$ implies $0 \leq S_{n} \leq T_{n}$. This finishes the proof. 


\section{Existence of invariant monotone couplings}

We shall now use the previous results to show the existence of certain $\Gamma$-invariant couplings between $\Gamma$-invariant determinantal measures on $\Gamma$ itself, as well as on the edge set of any Cayley graph of $\Gamma$. Recall that a positive contraction $Q$ in $R(\Gamma)$ leads to a $\Gamma$-invariant determinantal measure on $\Gamma$, which we denote by $\mathbf{P}^{Q}$. Note also that $\mathbf{P}^{Q_{2}}$ stochastically dominates $\mathbf{P}^{Q_{1}}$ if $0 \leq Q_{1} \leq Q_{2} \leq I$. This is stated in Theorem 2.1 in the finite case and is shown to imply the same for the infinite case in [37].

TheOREM 5.1. Let $\Gamma$ be a sofic group and $S$ be a finite generating set of elements in $\Gamma$. If $0 \leq Q_{1} \leq Q_{2} \leq I$ in $R(\Gamma)$ or in $R(\Gamma, S)$, then there exists a $\Gamma$-invariant (sofic) monotone coupling of $\mathbf{P}^{Q_{1}}$ and $\mathbf{P}^{Q_{2}}$.

Proof. Let $\left(G_{n}\right)_{n}$ be a sequence of finite $S$-labelled Schreier graphs whose limit is $G:=$ Cay $(\Gamma, S)$. We prove the theorem for operators in $R(\Gamma)$, as the other case is essentially identical. By Proposition 4.4 and Lemma 4.7, there exist $0 \leq S_{n} \leq T_{n} \leq I$ in $B\left(\ell^{2}\left(\mathrm{~V}_{n}\right)\right)$ so that $\left(S_{n}\right)_{n}$ and $\left(T_{n}\right)_{n}$ represent $\iota\left(Q_{1}\right)$ and $\iota\left(Q_{2}\right)$ in the ultraproduct. Let $\mu_{n}$ be a monotone coupling of $\mathbf{P}^{S_{n}}$ with $\mathbf{P}^{T_{n}}$, as obtained from Strassen's theorem [55]. As explained in [4, Example 10.3], the random weak limit of $\left(\mu_{n}\right)_{n}$ (perhaps after taking a subsequence) is a $\Gamma$ invariant coupling of $\mathbf{P}^{Q_{1}}$ and $\mathbf{P}^{Q_{2}}$, which is necessarily monotone. However, we give another proof in the framework we are using here.

Note that $\mu_{n}$ is a probability measure on $2^{\mathbb{V}_{n}} \times 2^{\mathrm{V}_{n}}$. In general, given a set $V$, let $V^{\prime}$ be a disjoint copy of $V$ with bijection $\phi: V \rightarrow V^{\prime}$ and identify elements of $2^{V} \times 2^{V}$ with subsets of $V \cup V^{\prime}$. Thus, elementary cylinder events are identified with events of the form $\mathcal{A}=\left\{A \subset V \cup V^{\prime} ; A \supset A_{1}, A \cap A_{2}=\varnothing\right\}$ for finite $A_{1}, A_{2} \subset V \cup V^{\prime}$. Let $A_{1}, A_{2} \subset \Gamma \cup \Gamma^{\prime}$. Let $\mathcal{A}:=\left\{A \subset \Gamma \cup \Gamma^{\prime} ; A \supset A_{1}, A \cap A_{2}=\varnothing\right\}$. Let $\sigma: \Gamma \rightarrow \mathbf{F}_{S}$ be a section of the natural surjection $\pi: \mathbf{F}_{S} \rightarrow \Gamma$. For $v \in \bigvee_{n}$, write

$$
A_{i, n, v}:=v \cdot \sigma\left(A_{i} \cap \Gamma\right) \cup \phi\left(v \cdot \sigma\left(A_{i} \cap \phi(\Gamma)\right)\right) \subset \mathrm{V}_{n} \cup \mathrm{V}_{n}^{\prime}
$$

for $i \in\{1,2\}$ and define

$$
\mathcal{A}_{v, n}:=\left\{A \in 2^{\mathrm{V}_{n}} \times 2^{\mathrm{V}_{n}} ; A_{1, n, v} \subset A, A_{2, n, v} \cap A=\varnothing\right\} \subset 2^{\mathrm{V}_{n}} \times 2^{\mathrm{V}_{n}} .
$$

We define

$$
\tilde{\mu}(\mathcal{A}):=\lim _{n \rightarrow \omega} \frac{1}{\left|\mathrm{~V}_{n}\right|} \sum_{v \in \mathrm{V}_{n}} \mu_{n}\left(\mathcal{A}_{v, n}\right) .
$$

Since ultralimits are finitely additive, this extends to define a consistent measure on cylinder events, whence by Kolmogorov's Extension Theorem, there exists a unique measure $\mu$ on the Borel $\sigma$-algebra of $2^{\Gamma} \times 2^{\Gamma}$ that extends $\tilde{\mu}$. We claim that $\mu$ is the desired coupling. It is a basic property of the formula in the definition of $\tilde{\mu}$ that the action of $\mathbf{F}_{S}$ on $G_{n}$ just 
permutes the summands of the right-hand side. Hence, we conclude that $\tilde{\mu}$ is $\Gamma$-invariant. Uniqueness of the extension in Kolmogorov's Extension Theorem implies that $\mu$ is also $\Gamma$ invariant. It follows from Lemma 4.5 that the marginals of $\mu$ are just $\mathbf{P}^{Q_{1}}$ and $\mathbf{P}^{Q_{2}}$. Finally, it is a monotone coupling because each $\mu_{n}$ is monotone. This finishes the proof.

As a special case, we obtain the following:

COROLlary 5.2. Let $\Gamma$ be a sofic group and $S$ be a finite generating set of elements in $\Gamma$. There exists a $\Gamma$-invariant monotone coupling between $\mathrm{WSF}_{G} \preccurlyeq \mathrm{FSF}_{G}$ on the associated Cayley graph.

PRoOF. It remains only to pass from the Cayley diagram to the Cayley graph. Every edge of the Cayley graph is doubled in the diagram. However, recall that in order to define the spanning forest measures, one must first choose an orientation for each unoriented edge. Thus, we may simply choose one of each pair in the diagram to be the orientation of the corresponding unoriented edge. If we ignore the other edge, then the corresponding determinantal probability measures are precisely the ones we want when we identify each chosen oriented edge with its corresponding unoriented edge.

The preceding corollary was proven by Bowen for residually amenable groups [9]. Elek and Szabó [21] gave examples of finitely generated sofic groups that are not residually amenable. Later, Cornulier gave the first examples of finitely presented groups that are sofic but not residually amenable (or even limits of amenable groups) [16, Corollary 3].

Similar reasoning shows, e.g., that if $0 \leq Q_{1} \leq \cdots \leq Q_{r} \leq I$ in $R(\Gamma)$, then there exists a $\Gamma$-invariant sofic coupling of all $\mathbf{P}^{Q_{i}}$ simultaneously that is monotone for each successive pair $i, i+1$.

\section{Free uniform spanning forest measures as limits over a sofic approximation}

For certain determinantal measures we can say more. Let us first introduce some more notation. Note that for $d \in \mathbb{N}$, the embedding $(R(\Gamma), \tau) \subset \prod_{n \rightarrow \omega}\left(B\left(\ell^{2} \bigvee_{n}\right), \operatorname{tr}_{\vee_{n}}\right)$ gives rise to an embedding

$$
\left(M_{d} R(\Gamma), \tau^{(d)}\right) \subset \prod_{n \rightarrow \omega}\left(M_{d} B\left(\ell^{2} \bigvee_{n}\right), \operatorname{tr}_{\mathrm{V}_{n}}^{(d)}\right)
$$

where $\operatorname{tr}_{\mathrm{V}_{n}}^{(d)}$ denotes the natural extension of the trace $\operatorname{tr}_{\mathrm{V}_{n}}$ to $M_{d} B\left(\ell^{2} \mathrm{~V}_{n}\right)$. Similarly, we denote the natural extension of $\tau$ to $M_{d} R(\Gamma)$ by $\tau^{(d)}$. Let $G=(\mathrm{V}, \mathrm{E})$ be an $S$-labelled Schreier graph. We shall consider the natural extension of $\rho_{G}$ (defined after (2)) to

$$
\rho_{G}^{(d)}: M_{d} \mathbb{C F}_{S} \rightarrow M_{d} B\left(\ell^{2} \mathrm{~V}\right)
$$


The following theorem is a variant of Lück's approximation theorem. In the generality that we need, it was first proved by Elek and Szabó in [20, Proposition 6.1(a)]. Let us state what we need in our notation.

THEOREM 6.1 (Elek-Szabó). Let $\Gamma$ be a sofic group and $S$ be a finite generating set of elements in $\Gamma$. Let $\left(G_{n}\right)_{n}$ be a sequence of finite $S$-labelled Schreier graphs whose limit is $G:=\operatorname{Cay}(\Gamma, S)$. Let $d \in \mathbb{N}$ and $a \in M_{d} \mathbb{Z} \mathbf{F}_{S}$. Let $P_{n} \in M_{d} B\left(\ell^{2} \bigvee_{n}\right)$ denote the projection onto the kernel of $\rho_{G_{n}}^{(d)}(a)$ and $P \in M_{d} R \Gamma$ denote the projection onto the kernel of $\rho_{G}^{(d)}(a)$. Then

$$
\lim _{n \rightarrow \infty} \operatorname{tr}_{\mathrm{V}_{n}}^{(d)}\left(P_{n}\right)=\tau^{(d)}(P) .
$$

The statement of the previous theorem is not just a consequence of weak ${ }^{*}$ convergence of spectral measures. The proof uses in an essential way the integrality of coefficients of $a \in M_{d} \mathbb{Z} \mathbf{F}_{S}$. The first results of this form were obtained by Lück in [35], and over the years they inspired many results of the same type. Analogues of Lück's approximation theorem in the context of convergent sequences of finite graphs were studied in [2].

Note that Proposition 6.1 in [20] contains a part (b), which asserted that

$$
\int_{0^{+}}^{\infty} \log (t) d \mu_{\left|\rho_{G_{n}}^{(d)}(a)\right|}(t) \rightarrow \int_{0^{+}}^{\infty} \log (t) d \mu_{\left|\rho_{G}^{(d)}(a)\right|}(t) \quad \text { as } n \rightarrow \infty,
$$

in a slightly more general form not assuming that the approximation is given by Schreier graphs; see [20]. This part of the claim remained unproven in [20]. Very recently, it was discovered that [20, Proposition 6.1(b)] is actually wrong (in the form more general than (6)). It was shown independently by Lovász and by Grabowski-Thom that already the Cayley diagram of $G=\mathbb{Z}$ with respect to some specific multi-set of generators admits a sofic approximation $\left(G_{n}\right)_{n}$-albeit not by Schreier graphs - so that $\left|\mathrm{V}\left(G_{n}\right)\right|^{-1} \log \left|\operatorname{det} A\left(G_{n}\right)\right|$ does not converge, where $A\left(G_{n}\right)$ denotes the adjacency matrix of $G_{n}$. It is still possible that (6) holds as written here.

COROllary 6.2. Let $\Gamma$ be a sofic group and $S$ be a finite generating set of elements in $\Gamma$. Let $\left(G_{n}\right)_{n}$ be a sequence of finite $S$-labelled Schreier graphs whose limit is $G:=\operatorname{Cay}(\Gamma, S)$. Let $d \in \mathbb{N}$ and $a \in M_{d} \mathbb{Z} \mathbf{F}_{S}$. Let $P_{n} \in M_{d} B\left(\ell^{2} \bigvee_{n}\right)$ denote the projection onto the kernel of $\rho_{G_{n}}^{(d)}(a)$ and $P \in M_{d} R \Gamma$ denote the projection onto the kernel of $\rho_{G}^{(d)}(a)$. Then the sequence $\left(P_{n}\right)_{n}$ represents $\iota_{S}(P) \in \prod_{n \rightarrow \omega}\left(M_{d} B\left(\ell^{2} \bigvee_{n}\right), \operatorname{tr}_{\mathrm{V}_{n}}^{(d)}\right)$.

Proof. Since $\operatorname{ker}\left(T^{*} T\right)=\operatorname{ker}(T)$ for any operator $T$ on a Hilbert space, we may assume without loss of generality that $a=b^{*} b$ for some $b \in M_{d} \mathbb{C F}_{s}$. We set $T_{n}:=\rho_{G_{n}}^{(d)}(a)$ and $T:=\iota_{S}\left(\rho^{(d)}(a)\right)$. It is clear from Corollary 4.4 that the sequence $\left(T_{n}\right)_{n}$ represents $T$. Set $c:=\sum_{i, j=1}^{d}\left\|a_{i, j}\right\|_{1}$, where $a_{i, j}$ denotes the $(i, j)$-entry of the matrix $a$ and $\left\|\sum_{\gamma} \alpha_{\gamma} \gamma\right\|_{1}:=$ $\sum_{\gamma}\left|\alpha_{\gamma}\right|$. It is easy to verify that $c \geq \sup \left\{\left\|T_{n}\right\| ; n \in \mathbb{N}\right\}$ and $c \geq\|T\|$. By Lemma 4.2, for 
any continuous function $f: \mathbb{R} \rightarrow \mathbb{R}$, the sequence $\left(f\left(T_{n}\right)\right)_{n}$ represents $f(T)$. Now, let $\left(f_{k}\right)_{k}$ be a sequence of polynomials that are non-negative on $[0, c]$ and satisfy

$$
\inf _{k} f_{k}(x)= \begin{cases}1 & \text { if } x=0 \\ 0 & \text { if } x \in(0, c]\end{cases}
$$

For example, one can take $f_{k}(x):=(1-x / c)^{2 k}$. Note that $\inf _{k} f_{k}\left(T_{n}\right)=P_{n}$ and $\inf _{k} f_{k}(T)=$ $\iota_{S}(P)$, where the infimum is taken with respect to the usual ordering on self-adjoint operators. It is clear from Lemma 4.2 that $\left(P_{n}\right)_{n}$ represents a projection that is smaller than $f_{k}(T)$ for each $k \in \mathbb{N}$ and hence smaller than $\iota_{S}(P)=\inf _{k} f_{k}(T)$. Lück's approximation theorem (Theorem 6.1) says that $\lim _{n \rightarrow \infty} \operatorname{tr}_{\mathrm{V}_{n}}^{(d)}\left(P_{n}\right)=\tau^{(d)}(P)=\tau_{\omega}^{(d)}\left(\iota_{S}(P)\right.$ ). This shows that the subprojection of $\iota_{S}(P)$ that $\left(P_{n}\right)_{n}$ represents has the same trace as $\iota_{S}(P)$. Since $\tau_{\omega}^{(d)}$ is faithful, this implies that $\left(P_{n}\right)_{n}$ represents $\iota_{S}(P)$. This finishes the proof.

Let $a \in M_{d} \mathbb{Z} \mathbf{F}_{S}$ be self-adjoint. The heart of the proof of the preceding theorem is that the convergence of spectral measures of $T_{n}:=\rho_{G_{n}}^{(d)}(a)$ to the spectral measure of $T:=\rho^{(d)}(a)$ is far better than expected from Remark 4.3, due to the integrality of the coefficients of $a$. Indeed, Lück's approximation theorem asserts that $\lim _{n \rightarrow \infty} \mu_{T_{n}}(\{0\})=\mu_{T}(\{0\})$, which is not a consequence of weak convergence $\mu_{T_{n}} \rightarrow \mu_{T}$ alone. In fact, even more is true. It is a consequence of results in [57] that the integrated densities $F_{T_{n}}(\lambda):=\mu_{T_{n}}((-\infty, \lambda])$ converge uniformly to $F_{T}(\lambda):=\mu_{T}((-\infty, \lambda])$, i.e., $\sup \left\{\left|F_{T_{n}}(\lambda)-F_{T}(\lambda)\right| ; \lambda \in \mathbb{R}\right\} \rightarrow 0$ as $n \rightarrow \infty$. This allows for more results like Theorem 6.2, for example, for other spectral projections of the operator $\rho^{(d)}(a)$.

Suppose that the random weak limit of $\left(G_{n}\right)$ is a Cayley graph $G$. In the case where $G$ is amenable and $G_{n}$ are merely connected subgraphs of $G$, we have that the random weak limit of the uniform spanning tree measures $\mathrm{UST}_{G_{n}}$ equals $\mathrm{WSF}_{G}=\mathrm{FSF}_{G}$. Despite the definition of FSF, the random weak limit of UST $_{G_{n}}$ need not be FSF $_{G}$ for a sofic approximation $\left(G_{n}\right)_{n}$ to a non-amenable group, $G$. In fact, the limit of UST $_{G_{n}}$ is always $\mathrm{WSF}_{G}$ (see [4, Proposition 7.1]). It is more complicated to get $\mathrm{FSF}_{G}$ as a limit. The proof of Corollary 5.2 provides such, but is not very explicit. Here we give a more explicit method, which still provides an invariant monotone coupling with $\mathrm{WSF}_{G}$. For $L \geq 0$, let $\diamond_{L}(G)$ denote the space spanned by the cycles in $G$ of length at most $L$. Write $\mathrm{FSF}_{G, L}$ for the determinantal probability measure corresponding to the projection onto $\diamond_{L}(G)^{\perp}$. This measure is not necessarily concentrated on forests; rather, it is concentrated on subgraphs of girth larger than L. By Theorem 2.1, we have $\mathrm{UST}_{G} \preccurlyeq \mathrm{FSF}_{G, L}$ for all finite $G$ and $L$.

THEOREM 6.3. If $G$ is a Cayley graph of a group $\Gamma$ and is the random weak limit of $\left(G_{n}\right)_{n}$, then if $L(n) \rightarrow \infty$ sufficiently slowly, the random weak limit of $\mathrm{FSF}_{G_{n}, L(n)}$ equals $\mathrm{FSF}_{G}$. 
A subsequence of monotone couplings witnessing $\mathrm{UST}_{G_{n}} \preccurlyeq \mathrm{FSF}_{G_{n}, L(n)}$ has a $\Gamma$-invariant monotone coupling witnessing $\mathrm{WSF}_{G} \preccurlyeq \mathrm{FSF}_{G}$ as a weak* limit.

Proof. By Corollary 6.2 and Lemma 4.5, for all $L \geq 0$, the random weak limit of $\mathrm{FSF}_{G_{n}, L}$ exists and equals $\mathrm{FSF}_{G, L}$. Since $\diamond_{L}(G) \uparrow \diamond(G)$, the weak* limit of $\mathrm{FSF}_{G, L}$ equals $\mathrm{FSF}_{G}$. A subsequence of monotone couplings witnessing $\mathrm{UST}_{G_{n}} \preccurlyeq \mathrm{FSF}_{G_{n}, L}$ has a $\Gamma$-invariant monotone coupling witnessing $\mathrm{WSF}_{G} \preccurlyeq \mathrm{FSF}_{G, L}$ as a random weak limit, which, as $L \rightarrow \infty$, has a $\Gamma$-invariant monotone coupling witnessing $\mathrm{WSF}_{G} \preccurlyeq \mathrm{FSF}_{G}$ as a weak* limit. The result follows.

In particular, with the assumptions of Theorem 6.3, calculation of average expected degree shows that

$$
\lim _{L \rightarrow \infty} \lim _{n \rightarrow \infty} \frac{\operatorname{dim} \diamond_{L}\left(G_{n}\right)}{\left|\mathrm{V}\left(G_{n}\right)\right|}=2 \beta_{1}^{(2)}(\Gamma)+2 .
$$

This equation is already known, as it follows from Lück's results and the Determinant Conjecture, which was established for sofic groups in [20, Theorem 5].

\section{Consequences of the invariant monotone couplings}

Given a network with positive edge weights and a time $t>0$, form the transition operator $P_{t}$ for continuous-time random walk whose rates are the edge weights; in the case of unbounded weights (or degrees), we take the minimal process, which dies after an explosion. That is, if the entries of a matrix $A$ indexed by the vertices are equal off the diagonal to the negative of the edge weights and the diagonal entries are chosen to make the row sums zero, then $P_{t}:=e^{-A t}$; in the case of unbounded weights, we take the selfadjoint extension of $A$ corresponding to the minimal process. The matrix $A$ is called the infinitesimal generator or the Laplacian of the network.

When the weights are random, we have a continuous-time random walk in a random environment. If the distribution $\mu$ of the edge weights is group-invariant, then $\mathbf{E}\left[P_{t}(o, o)\right]=$ $\operatorname{tr}_{\mu}\left(e^{-A t}\right)$. Hence, if there are two sets of random weights, $A^{(1)}$ and $A^{(2)}$, coupled by an invariant measure $\nu$ with the property that $A^{(1)}(e) \leq A^{(2)}(e) \nu$-a.s. for all edges $e$, then the corresponding return probabilities $P^{(1)}$ and $P^{(2)}$ satisfy $\mathbf{E}\left[P_{t}^{(1)}(o, o)\right] \geq \mathbf{E}\left[P_{t}^{(2)}(o, o)\right]$ : see $[4$, Theorem 5.1]. Whether this inequality holds without assuming the existence of an invariant coupling, but merely that the two weight distributions are invariant, is open; it was asked by $[24]$.

Of course, if the weights are simply the indicators of invariant random subsets, then we obtain random walk on the random clusters. Thus, when we have an invariant coupling of two percolation measures, we have the above inequality on return probabilities. In particular, we have shown that such an inequality holds when the two percolation measures are 
determinantal and arise from positive contractions in $R(\Gamma)$. A similar result holds when a more complicated increasing function of the random subsets is used (such as using for a weight of an edge the sum of the degrees of its endpoints in the cluster), since given an invariant monotone coupling of the two cluster measures, one easily constructs an invariant monotone coupling of such weights.

For another consequence of our coupling result, we consider the FSF. It was proved in [6] that for every Cayley graph, whether sofic or not, a.s. each tree in $\mathrm{WSF}_{G}$ has one end. In addition, [6] also proved that if $\mathrm{FSF}_{G} \neq \mathrm{WSF}_{G}$, then a.s. at least one tree in the FSF has infinitely many ends. [6] conjectured that a.s. every tree in the FSF has infinitely many ends in this case. We can now make a small contribution to this conjecture:

Corollary 7.1. If $G$ is the Cayley graph of a sofic group, then either $\mathrm{FSF}_{G}=\mathrm{WSF}_{G}$, in which case a.s. each tree in $\mathrm{FSF}_{G}$ has one end, or $\mathrm{FSF}_{G} \neq \mathrm{WSF}_{G}$, in which case a.s. each tree in $\mathrm{FSF}_{G}$ has one or infinitely many ends, with some tree having infinitely many ends.

Proof. Suppose that $\mathrm{FSF}_{G} \neq \mathrm{WSF}_{G}$. Let $\mu$ be an invariant monotone coupling of the two spanning forest measures. Because WSF is spanning, each tree in FSF consists of unions of (infinite) trees in WSF with additional connecting edges. If there are only finitely many connecting edges, say, $N$, in some tree $T$ in FSF, then each vertex in $T$ can send mass $1 / N$ to each endpoint of each of the connecting edges in $T$. Such endpoints would receive infinite mass, yet no point would send out mass more than 2. Thus, the Mass-Transport Principle tells us that this event has probability 0. Therefore, there are a.s. either no connecting edges or infinitely many in each tree of FSF. Combining this with what was previously known gives the corollary.

The above consequences of Theorem 5.1 were for specific models. We close with a general consequence that is relevant in ergodic theory.

For a set $X$, write $\pi_{x}:\{0,1\}^{X} \rightarrow\{0,1\}$ for the natural coordinate projections $(x \in X)$. For $K \subseteq X$, write $\mathscr{F}(K)$ for the $\sigma$-field on $\{0,1\}^{X}$ generated the maps $\pi_{x}$ for $x \in K$. When $X$ is the vertex set $\mathrm{V}$ of a graph, a probability measure $\mu$ on $\{0,1\}^{\vee}$ is called $m$-dependent if $\mathscr{F}\left(K_{1}\right), \ldots, \mathscr{F}\left(K_{p}\right)$ are independent whenever the sets $K_{i}$ are pairwise separated by graph distance $>m$. A similar definition holds when $X$ is the edge set of a graph. We say that $\mu$ is finitely dependent if it is $m$-dependent for some $m<\infty$.

Note that if $Q \in \mathbb{C} \Gamma$ is a positive contraction, then $\mathbf{P}^{Q}$ is finitely dependent. The Kaplansky density theorem implies that every positive contraction $Q \in R(\Gamma)$ is the limit in the strong operator topology (SOT) of positive contractions $Q_{n} \in \mathbb{C} \Gamma$ (see [28, Cor. 5.3.6]). Combining these two observations, we see that $\mathbf{P}^{Q}$ is the weak* limit of the finitely dependent measures $\mathbf{P}^{Q_{n}}$. Likewise, if $Q \in R(\Gamma, S)$ is a positive contraction, then there are positive 
contractions $Q_{n} \in M_{S}(\mathbb{C} \Gamma)$ such that $\mathbf{P}^{Q}$ is the weak* limit of the finitely dependent measures $\mathbf{P}^{Q_{n}}$.

In the case that $\Gamma$ is sofic, we can strengthen weak* convergence to $\bar{d}$-convergence because of our monotone coupling result. This follows ideas of [38], but that case, where $\Gamma$ is commutative, is much easier.

Let $\mu_{1}$ and $\mu_{2}$ be two $\Gamma$-invariant probability measures on $A^{W}$, where $\Gamma$ acts quasitransitively on $W$ and $A$ is finite. Let $W^{\prime}$ be a section of $\Gamma \backslash W$. Then Ornstein's $\bar{d}$-metric is defined as

$$
\bar{d}\left(\mu_{1}, \mu_{2}\right):=\min \left\{\sum_{w \in W^{\prime}} \mathbf{P}\left[X_{1}(w) \neq X_{2}(w)\right] ; X_{1} \sim \mu_{1}, X_{2} \sim \mu_{2},\left(X_{1}, X_{2}\right) \text { is } \Gamma \text {-invariant }\right\} .
$$

This is a metric for the following reason. Suppose that $\left(X_{1}, X_{2}\right)$ is a joining of $\left(\mu_{1}, \mu_{2}\right)$ and $\left(X_{3}, X_{4}\right)$ is a joining of $\left(\mu_{2}, \mu_{3}\right)$. Given a Borel set $C \subseteq A^{W}$, write $f_{C}\left(X_{2}\right):=\mathbf{P}\left[X_{1} \in C \mid X_{2}\right]$ and $g_{C}\left(X_{3}\right):=\mathbf{P}\left[X_{4} \in C \mid X_{3}\right]$. The relatively independent joining of $\left(X_{1}, X_{2}\right)$ and $\left(X_{3}, X_{4}\right)$ over $\mu_{2}$ is defined to be the measure $\mu$ on $\left(A^{W}\right)^{3}$ determined by

$$
\left(C_{1}, C_{2}, C_{3}\right) \mapsto \int_{C_{2}} f_{C_{1}}(y) g_{C_{3}}(y) d \mu_{2}(y)
$$

for $C_{1}, C_{2}, C_{3} \subseteq 2^{W}$. It is easily verified and well known that this measure $\mu$ is indeed $\Gamma$ invariant, and therefore a joining. (Intuitively, we merely choose $X_{2}=X_{3}$ to create this joining out of the original pair of joinings. More precisely, $X_{1}$ and $X_{4}$ are then chosen independently given $X_{2}$.) Now choose the joinings $\left(X_{1}, X_{2}\right)$ and $\left(X_{3}, X_{4}\right)$ to achieve the minima in the definition of $\bar{d}$. If $\left(Y_{1}, Y_{2}, Y_{3}\right) \sim \mu$, then $\bar{d}\left(\mu_{1}, \mu_{3}\right) \leq \sum_{w \in W^{\prime}} \mathbf{P}\left[Y_{1}(w) \neq\right.$ $\left.Y_{3}(w)\right] \leq \sum_{w \in W^{\prime}} \mathbf{P}\left[Y_{1}(w) \neq Y_{2}(w)\right]+\sum_{w \in W^{\prime}} \mathbf{P}\left[Y_{2}(w) \neq Y_{3}(w)\right]=\sum_{w \in W^{\prime}} \mathbf{P}\left[X_{1}(w) \neq\right.$ $\left.X_{2}(w)\right]+\sum_{w \in W^{\prime}} \mathbf{P}\left[X_{2}(w) \neq X_{3}(w)\right]=\bar{d}\left(\mu_{1}, \mu_{2}\right)+\bar{d}\left(\mu_{2}, \mu_{3}\right)$, as desired.

If $\left(\mu_{1}, \mu_{2}, \ldots, \mu_{n}\right)$ is a sequence of $\Gamma$-invariant probability measures on $A^{W}$ and $\mu_{k, k+1}$ is a joining of $\left(\mu_{k}, \mu_{k+1}\right)$ for each $k=1,2, \ldots, n-1$, then there is an associated relatively independent joining of all $n$ measures obtained by successively taking a relatively independent joining $\left(Y_{1}, Y_{2}, Y_{3}\right)$ of $\left(\mu_{1}, \mu_{2}\right)$ with $\left(\mu_{2}, \mu_{3}\right)$ over $\mu_{2}$, then the relatively independent joining of $\left(Y_{1}, Y_{2}, Y_{3}\right)$ with $\left(\mu_{3}, \mu_{4}\right)$ over $\mu_{3}$, where we regard $\left(Y_{1}, Y_{2}, Y_{3}\right) \in(A \times A)^{W} \times A^{W}$, etc. By taking a limit of such joinings, we can do the same for an infinite sequence of invariant measures on $A^{W}$ with given successive joinings.

In case $A=\{0,1\}$ and there is a monotone joining of $\mu_{1}$ and $\mu_{2}$, then such a joining may be used to calculate $\bar{d}\left(\mu_{1}, \mu_{2}\right)$ :

LEMMA 7.2. Let $\mu_{1}$ and $\mu_{2}$ be two $\Gamma$-invariant probability measures on $2^{W}$, where $\Gamma$ acts quasi-transitively on $W$. Let $W^{\prime}$ be a section of $\Gamma \backslash W$. If there is a monotone joining 
$\left(X_{1}, X_{2}\right)$ of $\left(\mu_{1}, \mu_{2}\right)$, then

$$
\bar{d}\left(\mu_{1}, \mu_{2}\right)=\sum_{w \in W^{\prime}}\left|\mathbf{P}\left[X_{1}(w)=0\right]-\mathbf{P}\left[X_{2}(w)=0\right]\right|=\sum_{w \in W^{\prime}} \mathbf{P}\left[X_{1}(w) \neq X_{2}(w)\right] .
$$

Suppose that in addition, $\mu_{3}$ and $\mu_{4}$ are two $\Gamma$-invariant probability measures on $2^{W}$ such that there are monotone joinings witnessing $\mu_{1} \preccurlyeq \mu_{3} \preccurlyeq \mu_{2}$ and $\mu_{1} \preccurlyeq \mu_{4} \preccurlyeq \mu_{2}$. Then $\bar{d}\left(\mu_{3}, \mu_{4}\right) \leq \bar{d}\left(\mu_{1}, \mu_{2}\right)$.

PRoOF. It is clear that any joining $\left(X_{1}, X_{2}\right)$ of $\left(\mu_{1}, \mu_{2}\right)$ has the property that

$$
\sum_{w \in W^{\prime}} \mathbf{P}\left[X_{1}(w) \neq X_{2}(w)\right] \geq \sum_{w \in W^{\prime}}\left|\mathbf{P}\left[X_{1}(w)=0\right]-\mathbf{P}\left[X_{2}(w)=0\right]\right|
$$

and that a monotone joining gives equality. Furthermore, if we extend $\left(X_{1}, X_{2}\right)$ to a relatively independent joining $\left(X_{1}, X_{2}, X_{3}, X_{4}\right)$ with the assumed joinings satisfying $X_{1} \leq X_{3} \leq X_{2}$ and $X_{1} \leq X_{4} \leq X_{2}$, then the joining $\left(X_{3}, X_{4}\right)$ witnesses the desired inequality.

We shall prove the following:

THEOREM 7.3. Let $\Gamma$ be a sofic group and $S$ be a finite generating set of elements in $\Gamma$. If $Q$ is a positive contraction in $R(\Gamma)$ or in $R(\Gamma, S)$, then there exists a sequence of positive contractions $Q_{n}$ in $\mathbb{C} \Gamma$ or in $M_{S}(\mathbb{C} \Gamma)$ such that the finitely dependent probability measures $\mathbf{P}^{Q_{n}}$ converge to $\mathbf{P}^{Q}$ in the $\bar{d}$-metric.

Note that when $\Gamma$ is amenable, Theorem 5.1 is easy. In addition, when $\Gamma$ is amenable, it is known that the $\Gamma$-invariant finitely dependent processes are isomorphic to Bernoulli shifts by using the very weak Bernoulli condition of [45], extended to the amenable case by [3]; that factors of Bernoulli shifts are isomorphic to Bernoulli shifts [44, 46]; and that the class of processes isomorphic to Bernoulli shifts is $\bar{d}$-closed [44, 46]. Thus, we have the following corollary, which was proved for abelian $\Gamma$ in [38]:

COROLlARY 7.4. Let $\Gamma$ be an amenable group and $S$ be a finite generating set of elements in $\Gamma$. If $Q$ is a positive contraction in $R(\Gamma)$ or in $R(\Gamma, S)$, then $\mathbf{P}^{Q}$ is isomorphic to a Bernoulli shift.

On the other hand, in the non-amenable setting, Popa gave an example of a factor of a Bernoulli shift that is not isomorphic to a Bernoulli shift. Indeed, [50, Corollary 2.14] showed that for any infinite group $\Gamma$ with Kazhdan's Property $(\mathrm{T})$, the natural action $\Gamma \curvearrowright\left(\mathbb{T}, \mu_{\text {Haar }}\right)^{\Gamma} /(\mathbb{Z} / n \mathbb{Z})$ is not isomorphic to a Bernoulli shift for any $n \geq 2$. Here, $\mathbb{Z} / n \mathbb{Z}$ is understood to act diagonally on $\left(\mathbb{T}, \mu_{\text {Haar }}\right)^{\Gamma}$ by rotation in the obvious way. For such $\Gamma$, it follows that the natural action $\Gamma \curvearrowright\left(\mathbb{Z} / n \mathbb{Z}, \mu_{\text {Haar }}\right)^{\Gamma} /(\mathbb{Z} / n \mathbb{Z})$ is not isomorphic to a Bernoulli shift for any $n \geq 2$.

Natural questions, therefore, include these, which are all settled in the amenable case: 
Question 7.5. Is every finitely dependent process a factor of a Bernoulli shift?

Question 7.6. Let $\Gamma$ act quasi-transitively on a countable set $W$ and let $A$ be finite. Is the class of measures on $A^{W}$ that are factors of Bernoulli shifts closed in the $\bar{d}$-metric?

Question 7.7. Are determinantal probability measures associated to equivariant positive contractions factors of Bernoulli shifts?

By Theorem 7.3, positive answers to Questions 7.5 and 7.6 would imply a positive answer to Question 7.7 on sofic groups.

In order to prove Theorem 7.3, we shall use two lemmas. We give statements and details for $R(\Gamma)$; they admit straightforward extensions to $R(\Gamma, S)=M_{S}(R(\Gamma)$ ).

LEMMA 7.8. If $Q$ and $Q^{\prime}$ are positive contractions in $R(\Gamma)$, then

$$
\bar{d}\left(\mathbf{P}^{Q}, \mathbf{P}^{Q^{\prime}}\right) \leq \frac{6\left\|Q-Q^{\prime}\right\|}{1+2\left\|Q-Q^{\prime}\right\|}
$$

Proof. Write $r:=\left\|Q-Q^{\prime}\right\|$ and $t:=r /(1+2 r)$. We set $Q_{t}:=(1-t) Q+t(I-Q)$. Then $Q \geq(1-t) Q$ and $Q_{t} \geq(1-t) Q$, whence by the triangle inequality and Lemma 7.2, we have

$$
\bar{d}\left(\mathbf{P}^{Q}, \mathbf{P}^{Q t}\right) \leq \bar{d}\left(\mathbf{P}^{Q}, \mathbf{P}^{(1-t) Q}\right)+\bar{d}\left(\mathbf{P}^{(1-t) Q}, \mathbf{P}^{Q t}\right) \leq\|t Q\|+\|t(I-Q)\| \leq 2 t .
$$

Likewise, with $Q_{t}^{\prime}:=(1-t) Q^{\prime}+t\left(I-Q^{\prime}\right)$, we have

$$
\bar{d}\left(\mathbf{P}^{Q^{\prime}}, \mathbf{P}^{Q_{t}^{\prime}}\right) \leq 2 t
$$

In addition, $t I \leq Q_{t}^{\prime} \leq(1-t) I$ and $Q_{t}-Q_{t}^{\prime}=(1-2 t)\left(Q-Q^{\prime}\right)$ has norm $(1-2 t) r=t$, whence

$$
0 \leq Q_{t}^{\prime}-t I \leq Q_{t} \leq Q_{t}^{\prime}+t I \leq I
$$

Lemma 7.2 again yields

$$
\bar{d}\left(\mathbf{P}^{Q_{t}}, \mathbf{P}^{Q_{t}^{\prime}}\right) \leq \bar{d}\left(\mathbf{P}^{Q_{t}^{\prime}-t I}, \mathbf{P}^{Q_{t}^{\prime}+t I}\right)=2 t .
$$

Putting together these inequalities and using the triangle inequality for the $\bar{d}$-metric gives $\bar{d}\left(\mathbf{P}^{Q}, \mathbf{P}^{Q^{\prime}}\right) \leq 6 t$, which is the desired result.

When $Q$ and $Q^{\prime}$ commute, one can improve the bound in Lemma 7.8 by replacing the norm on the right-hand side with the Schatten 1-norm. Recall that this norm is

$$
\|T\|_{1}:=\tau\left(\left(T^{*} T\right)^{1 / 2}\right) .
$$

In this language, when $\Gamma$ is abelian, [38] showed that $\bar{d}\left(\mathbf{P}^{Q}, \mathbf{P}^{Q^{\prime}}\right) \leq\left\|Q-Q^{\prime}\right\|_{1}$. In fact, the same proof can be adapted for all $\Gamma$ to the case that $Q$ and $Q^{\prime}$ commute. We do not know whether this inequality always holds, but we have the following weaker version: 
LEMmA 7.9. If $Q$ and $Q^{\prime}$ are positive contractions in $R(\Gamma)$, then

$$
\bar{d}\left(\mathbf{P}^{Q}, \mathbf{P}^{Q^{\prime}}\right) \leq 6 \cdot 3^{2 / 3}\left\|Q-Q^{\prime}\right\|_{1}^{1 / 3} .
$$

If $Q_{n}$ and $Q$ are positive contractions in $R(\Gamma)$ with $Q_{n} \rightarrow Q$ in $\mathrm{SOT}$, then $\bar{d}\left(\mathbf{P}^{Q_{n}}, \mathbf{P}^{Q}\right) \rightarrow 0$.

Proof. We shall use the Schatten 2-norm, $\|T\|_{2}:=\sqrt{\tau\left(T^{*} T\right)}$, and the Powers-Størmer inequality, $\left\|T_{1}-T_{2}\right\|_{2}^{2} \leq\left\|T_{1}^{2}-T_{2}^{2}\right\|_{1}$ for $0 \leq T_{1}, T_{2} \in R(\Gamma)$; see [12, Proposition 6.2.4] for a proof that extends to our context.

Write $T:=Q^{1 / 2}$ and $T^{\prime}:=\left(Q^{\prime}\right)^{1 / 2}$. Let $E$ be the spectral resolution of the identity for $T-T^{\prime}$, so that

$$
T-T^{\prime}=\int_{-1}^{1} s d E(s) .
$$

Thus,

$$
r:=\int_{-1}^{1} s^{2} d \nu(s)=\left\|T-T^{\prime}\right\|_{2}^{2} \leq\left\|Q-Q^{\prime}\right\|_{1}
$$

for the scalar measure $A \mapsto \nu(A):=\tau(E(A))$. Define $t:=(r / 3)^{1 / 3}$ and $B:=[-1,-t] \cup[t, 1]$. We have $\nu(B) \leq r / t^{2}$ by Markov's inequality. Write $P:=E(B)$ and $P^{\perp}:=E((-t, t))$. Define $Q_{1}:=T P T$ and $Q_{1}^{\prime}:=T^{\prime} P T^{\prime}$, and write $Q_{2}:=Q-Q_{1}, Q_{2}^{\prime}:=Q^{\prime}-Q_{1}^{\prime}$. These are all positive contractions in $R(\Gamma)$; for example, $Q_{2}=T P^{\perp} T=\left(P^{\perp} T\right)^{*}\left(P^{\perp} T\right) \geq 0$. Furthermore,

$$
Q_{2}-Q_{2}^{\prime}=T P^{\perp}\left(T-T^{\prime}\right)+\left(T-T^{\prime}\right) P^{\perp} T^{\prime}
$$

and $\|T\|,\left\|T^{\prime}\right\| \leq 1$, whence $\left\|Q_{2}-Q_{2}^{\prime}\right\| \leq 2\left\|\left(T-T^{\prime}\right) P^{\perp}\right\|$. Since

$$
\left(T-T^{\prime}\right) P^{\perp}=\int_{(-t, t)} s d E(s)
$$

it follows that $\left\|\left(T-T^{\prime}\right) P^{\perp}\right\| \leq t$, whence $\left\|Q_{2}-Q_{2}^{\prime}\right\| \leq 2 t$ and $\bar{d}\left(\mathbf{P}^{Q_{2}}, \mathbf{P}^{Q_{2}^{\prime}}\right) \leq 12 t$ by Lemma 7.8. Now, $\tau\left(Q_{1}\right)=\tau\left(T^{2} P\right)=\tau\left(T^{2} P^{2}\right)=\tau\left(P T^{2} P\right)=\left\|T P \delta_{o}\right\|^{2} \leq\|T\|^{2}\left\|P \delta_{o}\right\|^{2} \leq$ $\tau(P)=\nu(B) \leq r / t^{2}$. Since $Q_{2} \leq Q$, it follows that $\bar{d}\left(\mathbf{P}^{Q}, \mathbf{P}^{Q_{2}}\right)=\tau\left(Q-Q_{2}\right)=\tau\left(Q_{1}\right) \leq r / t^{2}$. Likewise, $\bar{d}\left(\mathbf{P}^{Q^{\prime}}, \mathbf{P}^{Q_{2}^{\prime}}\right) \leq r / t^{2}$. Therefore,

$$
\bar{d}\left(\mathbf{P}^{Q}, \mathbf{P}^{Q^{\prime}}\right) \leq \bar{d}\left(\mathbf{P}^{Q}, \mathbf{P}^{Q_{2}}\right)+\bar{d}\left(\mathbf{P}^{Q_{2}}, \mathbf{P}^{Q_{2}^{\prime}}\right)+\bar{d}\left(\mathbf{P}^{Q_{2}^{\prime}}, \mathbf{P}^{Q^{\prime}}\right) \leq \frac{r}{t^{2}}+12 t+\frac{r}{t^{2}}=6(9 r)^{1 / 3}
$$

as desired.

The second part of the assertion follows from the inequality

$$
\left\|Q_{n} \delta_{o}-Q \delta_{o}\right\|=\left\|Q_{n}-Q\right\|_{2} \geq\left\|Q_{n}-Q\right\|_{1}
$$

This finishes the proof.

We remark that if it is assumed only that $Q_{n}$ converges to $Q$ in WOT, then it does not follow that $\mathbf{P}^{Q_{n}}$ converges to $\mathbf{P}^{Q}$ in $\bar{d}$. In fact, on $\mathbb{Z}$, entropies need not converge: see the end 
of Sec. 6 of [38] for examples. Here, we are using the fact that for processes on $\mathbb{Z}$, entropy is $\bar{d}$-continuous [25, Proposition 15.20].

Proof of Theorem 7.3: This is immediate from Lemma 7.9 and Kaplansky's Density Theorem, i.e., the fact that positive contractions in $R(\Gamma)$ are SOT-limits of positive contractions in $\mathbb{C} \Gamma$.

By analogy with Bernoulli processes in the amenable case, one could ask whether determinantal processes are finitely determined, where we could define $\mu$ as finitely determined if whenever $\mu_{n} \rightarrow \mu$ weak w $^{*}$ and in sofic entropy, we have $\bar{d}\left(\mu_{n}, \mu\right) \rightarrow 0$. The converse presumably holds for all processes. When $\Gamma$ is amenable, this is known since sofic entropy equals ordinary metric entropy. Here, we are relying on definitions and results of [10].

Numerical calculation suggests that the inequality $\bar{d}\left(\mathbf{P}^{Q}, \mathbf{P}^{Q^{\prime}}\right) \leq\left\|Q-Q^{\prime}\right\|_{1}$ always holds, even for finite matrices without any invariance. Our proof of the weaker inequality Lemma 7.9 holds in that generality. These inequalities appear to imply similar inequalities for continuous determinantal point processes $(\mathscr{X}, \mathscr{Y})$, where the $\bar{d}$-metric is replaced by taking the minimum over all joinings of the intensity of the symmetric difference $\mathscr{X} \triangle \mathscr{Y}$; we plan to pursue this elsewhere.

\section{Unimodular random rooted graphs}

We now extend the preceding theorems to their natural setting encompassing all random weak limits of finite graphs with bounded degree (and somewhat beyond). One other setting in which it would be natural to investigate these questions is that of vertex-transitive graphs and their automorphism-invariant determinantal probability measures. However, we are able to treat only the sofic ones (again), which, in particular, excludes all non-unimodular transitive graphs.

We review a few definitions from the theory of unimodular random rooted networks; for more details, see [4]. A network is a (multi-)graph $G=(\mathrm{V}, \mathrm{E})$ together with a complete separable metric space $\Xi$ called the mark space and maps from $V$ and $E$ to $\Xi$. Images in $\Xi$ are called marks. The only assumption on degrees is that they are finite when loops are not counted. We omit the mark maps from our notation for networks.

A rooted network $(G, o)$ is a network $G$ with a distinguished vertex of $G$, called the root. A rooted isomorphism of rooted networks is an isomorphism of the underlying networks that takes the root of one to the root of the other. We do not distinguish between a rooted network and its isomorphism class. Let $\mathcal{G}_{*}$ denote the set of rooted isomorphism classes of rooted connected locally finite networks. Define a separable complete metric $d_{*}: \mathcal{G}_{*} \times \mathcal{G}_{*} \rightarrow[0,1]$ on $\mathcal{G}_{*}$ by letting the distance between $\left(G_{1}, o_{1}\right)$ and $\left(G_{2}, o_{2}\right)$ be 
$1 /(1+\alpha)$, where $\alpha$ is the supremum of those $r>0$ such that there is some rooted isomorphism of the balls of (graph-distance) radius $\lfloor r\rfloor$ around the roots of $G_{i}$ such that each pair of corresponding marks has distance less than $1 / r$. For probability measures $\mu, \mu_{n}$ on $\mathcal{G}_{*}$, we write $\mu_{n} \Rightarrow \mu$ when $\mu_{n}$ converges weakly with respect to this metric.

For a (possibly disconnected) network $G$ and a vertex $x \in \mathrm{V}(G)$, write $G_{x}$ for the connected component of $x$ in $G$. If $G$ is finite, then write $U_{G}$ for a uniform random vertex of $G$ and $U(G)$ for the corresponding distribution of $\left(G_{U_{G}}, U_{G}\right)$ on $\mathcal{G}_{*}$. Suppose that $G_{n}$ are finite networks and that $\mu$ is a probability measure on $\mathcal{G}_{*}$. We say that the random weak limit of a sequence $\left(G_{n}\right)_{n}$ is $\mu$ if $U\left(G_{n}\right) \Rightarrow \mu$.

A probability measure that is a random weak limit of finite networks is called sofic. In particular, a group is called sofic when its Cayley diagram is sofic.

All sofic measures are unimodular, which we now define. Similarly to the space $\mathcal{G}_{*}$, we define the space $\mathcal{G}_{* *}$ of isomorphism classes of locally finite connected networks with an ordered pair of distinguished vertices and the natural topology thereon. We shall write a function $f$ on $\mathcal{G}_{* *}$ as $f(G, x, y)$. We refer to $f(G, x, y)$ as the mass sent from $x$ to $y$ in $G$.

DEFINITION 8.1. Let $\mu$ be a probability measure on $\mathcal{G}_{*}$. We call $\mu$ unimodular if it obeys the Mass-Transport Principle: For all Borel $f: \mathcal{G}_{* *} \rightarrow[0, \infty]$, we have

$$
\int \sum_{x \in \mathrm{V}(G)} f(G, o, x) d \mu(G, o)=\int \sum_{x \in \mathrm{V}(G)} f(G, x, o) d \mu(G, o) .
$$

It is easy to see that every sofic measure is unimodular, as observed by [7], who introduced this general form of the Mass-Transport Principle under the name "intrinsic Mass-Transport Principle". The converse was posed as a question by [4]; it remains open.

Consider the Hilbert space $\mathscr{H}(\mu):=\int^{\oplus} \ell^{2}(\mathrm{~V}(G)) d \mu(G, o)$, a direct integral (see, e.g., [43] or [29, Chapter 14]). Here, we always choose canonical representatives for rootedisomorphism classes of networks, as explained in [4, Sec. 2]; in particular, $\mathrm{V}(G)=\mathbb{N}$. However, this is merely for technical reasons of measurability, so we omit this from our notation. The space $\mathscr{H}(\mu)$ is defined as the set of ( $\mu$-equivalence classes of) $\mu$-measurable functions $\xi$ defined on (canonical) rooted networks $(G, o)$ that satisfy $\xi(G, o) \in \ell^{2}(\mathrm{~V}(G))$ and $\int\|\xi(G, o)\|^{2} d \mu(G, o)<\infty$. We write $\xi=\int^{\oplus} \xi(G, o) d \mu(G, o)$. The inner product is given by $\langle\xi, \eta\rangle:=\int\left\langle\xi(G, o), \eta(G, o\rangle d \mu(G, o)\right.$. Let $T:(G, o) \mapsto T_{G, o}$ be a measurable assignment of bounded linear operators on $\ell^{2}(\mathrm{~V}(G))$ with $\mu$-finite supremum of the norms $\left\|T_{G, o}\right\|$. Then $T$ induces a bounded linear operator $T:=T^{\mu}:=\int^{\oplus} T_{G, o} d \mu(G, o)$ on $\mathscr{H}$ via

$$
T^{\mu}: \int^{\oplus} \xi(G, o) d \mu(G, o) \mapsto \int^{\oplus} T_{G, o} \xi(G, o) d \mu(G, o)
$$

The norm $\left\|T^{\mu}\right\|$ of $T^{\mu}$ is the $\mu$-essential supremum of $\left\|T_{G, o}\right\|$. We say that $T$ as above is equivariant if for all network isomorphisms $\phi: G_{1} \rightarrow G_{2}$ preserving the marks, all $o_{1}, x, y \in$ 
$\mathrm{V}\left(G_{1}\right)$ and all $o_{2} \in \mathrm{V}\left(G_{2}\right)$, we have $\left\langle T_{G_{1}, o_{1}} \delta_{x}, \delta_{y}\right\rangle=\left\langle T_{G_{2}, o_{2}} \delta_{\phi(x)}, \delta_{\phi(y)}\right\rangle$. For $T \in B(\mathscr{H}(\mu))$ equivariant, we have in particular that $T_{G, o}$ depends on $G$ but not on the root $o$, so we shall simplify our notation and write $T_{G}$ in place of $T_{G, o}$. For simplicity, we shall even write $T$ for $T_{G}$ when no confusion can arise.

We shall show that sofic probability measures can be extended to sofic measures on $S$ labelled Schreier networks; any new loops will get new marks indicating that they were not in the original underlying graph. This will be an important technical tool. It can only enlarge the class of equivariant operators.

Proposition 8.2. Let $\left(G_{n}\right)_{n}$ be networks with finitely many vertices and edges whose random weak limit is $\mu$ and whose mark space is $\Xi$. Let $|S|$ be at least twice the degree of every vertex in every $G_{n}$; possibly $|S|=\infty$. Then there exist $S$-labelled Schreier networks $H_{n}$ with mark space $\Xi \times\{0,1\}$ with the following properties:

(i) The underlying graph of each component of $H_{n}$ is equal to that of $G_{n}$ except that $H_{n}$ may have additional loops whose second mark coordinate is 1.

(ii) The first coordinate marks of each component of $H_{n}$ restricted to the underlying graph of $G_{n}$ agree with the marks on $G_{n}$.

(iii) The sequence $\left(H_{n}\right)_{n}$ has a random weak limit carried by S-labelled Schreier networks.

ProOF. Given a locally finite network $G$ with mark space $\Xi$, produce a random $S$-labelled Schreier network, $\phi(G)$, with mark space $\Xi \times\{0,1\}$ as follows. Let $\xi_{0}$ be an arbitrary element of $\Xi$. Let $U_{k}(e)$ be independent uniform $[0,1]$ random variables for $k \geq 1$ and $e \in \mathrm{E}(G)$. For an edge $e$, let $N(e)$ be the set of edges (including $e$ ) that share an endpoint with $e$. Write $S=\left\{s_{1}, s_{2}, \ldots\right\}$. We shall use the identity map for the involution $i: S \rightarrow S$. Assign a second mark coordinate of 0 to every vertex and edge of $G$. Assign the label $s_{1}$ to every edge $e$ such that $U_{1}(e)=\min \left\{U_{1}\left(e^{\prime}\right) ; e^{\prime} \in N(e)\right\}$. We assign further labels from $S$ recursively. Supposing that a partial assignment has been made using the random fields $U_{1}, \ldots, U_{k}$, let $J(e)$ be the minimum index $j$ such that no edge in $N(e)$ has been assigned the label $s_{j}$, if any. By choice of $|S|$, there is always such an index when $e$ does not yet have a label. Now assign the label $s_{J(e)}$ to every $e$ that does not have a label and for which $U_{k+1}(e)=\min \left\{U_{k+1}\left(e^{\prime}\right) ; e^{\prime} \in N(e)\right\}$. After all these assignments are completed, to every vertex $x$, add new loops with mark $\left(\xi_{0}, 1\right)$ so that the degree of $x$ is equal to $|S|$ and so that the resulting network, $\phi(G)$, is $S$-labelled.

Except for the fact that $\phi\left(G_{n}\right)$ is random, the sequence $\left(\phi\left(G_{n}\right)\right)_{n}$ has all the desired properties: note that $U\left(\phi\left(G_{n}\right)\right) \Rightarrow \nu$, where for a measurable set $A$ of rooted networks,

$$
\nu(A):=\int \mathbf{P}[(\phi(G), o) \in A] d \mu(G, o) .
$$

To fix this problem, let $\xi_{1}, \xi_{2}, \ldots$ be a dense subset of $\Xi$. Let $\psi_{n}: \Xi \rightarrow\left\{\xi_{1}, \ldots, \xi_{n}\right\}$ be a map that takes each mark $\xi$ to one of the closest points to it among $\left\{\xi_{1}, \ldots, \xi_{n}\right\}$. Then 
$\psi_{n}$ naturally induces a map $\tilde{\psi}_{n}$ on networks. The push-forward $\nu_{n}$ of the law of $U\left(\phi\left(G_{n}\right)\right)$ by $\tilde{\psi}_{n}$ gives a finitely supported probability measure. By taking a rational approximation of its probabilities, we may find a finite (disconnected) network $H_{n}$ such that $U\left(H_{n}\right)$ is within total variation distance $1 / n$ of $\nu_{n}$. This is the sequence desired.

Let $(X, d)$ be a separable compact metric space. From now on, we shall assume that $G=(\mathrm{V}, \mathrm{E})$ is a rooted connected $S$-labelled Schreier network. Moreover, we assume that the vertex labels take values in the space $X$. If $S$ is finite, then the space of marks $\Xi=S \cup X$ is compact. We denote the set of such (rooted connected $S$-labelled Schreier) networks by $\mathcal{G}_{\star}$ or $\mathcal{G}_{\star}(S, X)$. We use the following metric on $\mathcal{G}_{\star}(S, X)$ : Write $S=\left\{s_{1}, s_{2}, \ldots\right\}$. For a rooted $S$-labelled Schreier network $(G, o)$ and $n \geq 1$, let $G^{n}$ denote the connected component of $o$ in the subnetwork of $G$ formed by deleting all edges with a label $s_{k}$ (in either direction) for any $k>n$. Define a separable complete metric $d_{*}: \mathcal{G}_{\star} \times \mathcal{G}_{\star} \rightarrow[0,1]$ on $\mathcal{G}_{\star}$ by letting the distance between $\left(G_{1}, o_{1}\right)$ and $\left(G_{2}, o_{2}\right)$ be $1 /(1+\alpha)$, where $\alpha$ is the supremum of those $r>0$ such that there is some rooted isomorphism of the balls of (graph-distance) radius $\lfloor r\rfloor$ around the roots of $G_{i}^{\lfloor r\rfloor}$ that preserves marks up to an error of at most $1 / r$ in the metric of the mark space. Even if $S$ is infinite, $\mathcal{G}_{\star}(S, X)$ is a compact metric space (basically because $\{0,1\}^{S}$ is compact) and thus for any sequence $\left(\mu_{n}\right)_{n}$ of probability measures on $\mathcal{G}_{\star}(S, X)$, we have $\mu_{n} \Rightarrow \mu$ if and only if $\mu_{n} \rightarrow \mu$ in the weak* topology. For simplicity of notation, we omit all other edge marks; one may actually encode edge marks via vertex marks in any case.

Before we proceed, let us discuss a natural example. Let $\Gamma$ be a group that acts by homeomorphisms on a compact metrizable space $X$, preserving a probability measure, $\mu$. We assume that $\Gamma$ is generated by a finite symmetric set $S \subset \Gamma$ and define the involution $i: S \rightarrow S$ by $i(s)=s^{-1}$. Then we can associate to each point $x \in X$ the $S$-labelled rooted Schreier graph that arises from the restriction of the action of $\Gamma$ to the orbit of $x$. Each vertex in this graph carries a natural label in $X$. We obtain a continuous map $\varphi: X \rightarrow \mathcal{G}_{\star}(S, X)$ and can consider the push-forward measure $\varphi_{*}(\mu)$. This measure is unimodular. Moreover, there is a natural equivariant factor map $\psi: \mathcal{G}_{\star}(S, X) \rightarrow X$, sending a rooted graph to the label of its root.

Suppose that $\mu$ is a unimodular probability measure on $\mathcal{G}_{*}(S, X)$. Note that there is a continuous action of $\mathbf{F}_{S}$ on the compact space $\mathcal{G}_{\star}(S, X)$ that moves the root according to the labels seen at the root. Moreover, this action preserves the measure $\mu$, since $\mu$ is unimodular. Consider the ring $C\left(\mathcal{G}_{\star}(S, X)\right)$ of continuous functions on $\mathcal{G}_{\star}(S, X)$ and the algebraic crossed product algebra $C\left(\mathcal{G}_{\star}(S, X)\right) \rtimes \mathbf{F}_{S}$. Recall that $C\left(\mathcal{G}_{\star}(S, X)\right) \rtimes \mathbf{F}_{S}$ is a $*$-algebra and consists of finite formal sums $\sum_{w \in \mathbf{F}_{S}} f_{w} w$ with $f_{w} \in C\left(\mathcal{G}_{\star}(S, X)\right)$. The multiplication and involution are defined by linearity and the formulas

$$
\forall f_{1}, f_{2} \in C\left(\mathcal{G}_{\star}(S, X)\right) \quad \forall w_{1}, w_{2} \in \mathbf{F}_{S} \quad\left(f_{1} w_{1}\right) \cdot\left(f_{2} w_{2}\right):=f_{1}\left({ }^{w_{1}} f_{2}\right) w_{1} w_{2},
$$




$$
\left(f_{1} w_{1}\right)^{*}:=\left({ }^{w_{1}^{-1}} \bar{f}_{1}\right) w_{1}^{-1}
$$

where we use the convention ${ }^{w} f(G, v):=f(G, v \cdot w)$. The measure $\mu$ gives rise to a functional $\tau_{\mu}$ on the crossed product algebra as follows:

$$
\tau_{\mu}\left(\sum_{w \in \mathbf{F}_{S}} f_{w} w\right):=\sum_{w \in \mathbf{F}_{S}} \int \mathbf{1}_{o . w=o} \cdot f_{w}(G, o) d \mu(G, o) .
$$

There are two natural actions, $F$ and $M$, on $\mathscr{H}(\mu)$ of the algebra of continuous functions on $\mathcal{G}_{\star}(S, X)$, which will both be of importance. First of all, $f \in C\left(\mathcal{G}_{\star}(S, X)\right)$ can act as a constant on the fibers, i.e., $F(f)_{G, o}:=f(G, o) \cdot I_{\ell^{2}(\mathrm{~V}(G))}$, or equivalently

$$
F(f)(\xi):=\int^{\oplus} f(G, o) \xi(G, o) d \mu(G, o) .
$$

It is a basic fact that an operator $T \in B(\mathscr{H}(\mu))$ arises as above from a measurable family $(G, o) \mapsto T_{G, o}$ iff $T$ commutes with $F\left(C\left(\mathcal{G}_{\star}(S, X)\right)\right.$. A second action of $C\left(\mathcal{G}_{\star}(S, X)\right)$ is defined by the formula $M(f)_{G, o} \delta_{v}=f(G, v) \cdot \delta_{v}$ for all $v \in \mathrm{V}(G)$, in other words,

$$
M(f)(\xi):=\int^{\oplus} M(f)_{G, o} \xi(G, o) d \mu(G, o),
$$

where $M(f)_{G, o} \xi(G, o)(v):=f(G, v) \cdot \xi(G, o)(v)$.

We denote by $\rho_{\mu}(s) \in B(\mathscr{H}(\mu))$ the operator that assigns to $(G, o)$ the unitary operator on $\ell^{2}(\mathrm{~V}(G))$ that sends $\delta_{v}$ to $\delta_{v \cdot i(s)}$. It is easy to see that $\rho_{\mu}(s)$ is equivariant for all $s \in S$, and that this assignment extends to a unitary representation $\rho_{\mu}: \mathbf{F}_{S} \rightarrow U(\mathscr{H}(\mu))$ such that $\rho_{\mu}(s)^{*}=\rho_{\mu}(i(s))$ for all $s \in S$. This unitary representation $\rho_{\mu}: \mathbf{F}_{S} \rightarrow U(\mathscr{H}(\mu))$ extends in turn to a natural $*$-homomorphism $\rho_{\mu}: C\left(\mathcal{G}_{\star}(S, X)\right) \rtimes \mathbf{F}_{S} \rightarrow B(\mathscr{H}(\mu))$. Indeed, consider the natural representation of $C\left(\mathcal{G}_{\star}(S, X)\right)$ on $\mathscr{H}(\mu)$ by multiplication, $f \mapsto M(f)$. In order to see that $M$ and $\rho_{\mu}: \mathbf{F}_{S} \rightarrow U(\mathscr{H}(\mu))$ combine via linearity and $\rho_{\mu}(f w):=M(f) \rho_{\mu}(w)$ to a $*$-representation of the algebraic crossed product, it suffices to check that

$$
\forall w \in \mathbf{F}_{S}, f \in C\left(\mathcal{G}_{\star}(S, X)\right) \quad \rho_{\mu}(w) M(f) \rho_{\mu}(w)^{*}=M\left({ }^{w} f\right),
$$

as a simple verification using the definition of $C\left(\mathcal{G}_{\star}(S, X)\right) \rtimes \mathbf{F}_{S}$ shows. To prove that this equation indeed holds, we compute in $\ell^{2}(\mathrm{~V}(G))$ for $v \in \mathrm{V}(G)$ that

$$
\rho_{\mu}(w) M(f) \rho_{\mu}(w)^{*} \delta_{v}=\rho_{\mu}(w) M(f) \delta_{v . w}=f(G, v . w) \cdot \rho_{\mu}(w) \delta_{v . w}=M\left({ }^{w} f\right) \delta_{v} .
$$

This shows that $\rho_{\mu}: C\left(\mathcal{G}_{\star}(S, X)\right) \rtimes \mathbf{F}_{S} \rightarrow B(\mathscr{H}(\mu))$ exists as desired.

Now, if $\delta^{\mu} \in \mathscr{H}(\mu)$ denotes the naturally defined vector $(G, o) \mapsto \delta_{o} \in \ell^{2}(\mathrm{~V}(G))$, then

$$
\forall T \in C\left(\mathcal{G}_{\star}(S, X)\right) \rtimes \mathbf{F}_{S} \quad \tau_{\mu}(T)=\left\langle\rho_{\mu}(T) \delta^{\mu}, \delta^{\mu}\right\rangle .
$$


Indeed, for all $w \in \mathbf{F}_{S}, f \in C\left(\mathcal{G}_{\star}(S, X)\right)$, we have

$$
\begin{aligned}
\left\langle\rho_{\mu}(f w) \delta^{\mu}, \delta^{\mu}\right\rangle & =\left\langle M(f) \rho(w) \delta^{\mu}, \delta^{\mu}\right\rangle \\
& =\int\left\langle M(f) \rho(w) \delta_{o}, \delta_{o}\right\rangle d \mu(G, o) \\
& =\int\left\langle M(f) \delta_{o . w^{-1}}, \delta_{o}\right\rangle d \mu(G, o) \\
& =\int \mathbf{1}_{o . w=o} \cdot f(G, o) d \mu(G, o) .
\end{aligned}
$$

This shows that $\tau_{\mu}: C\left(\mathcal{G}_{\star}(S, X)\right) \rtimes \mathbf{F}_{S} \rightarrow \mathbb{C}$ is a positive linear functional. Although we shall not use it, we remark that the Hilbert space $\mathscr{H}(\mu)$ is the GNS-construction associated with the trace $\tau_{\mu}$; see [17, Lemma 4 in Chapter 4] for basics about the GNS-construction. We denote by $R(\mu)$ the von Neumann algebra generated by the $\rho_{\mu}$-image of $C\left(\mathcal{G}_{\star}(S, X)\right) \rtimes \mathbf{F}_{S}$, i.e., $R(\mu):=\rho_{\mu}\left(C\left(\mathcal{G}_{\star}(S, X)\right) \rtimes \mathbf{F}_{S}\right)^{\prime \prime}$. We call $R(\mu)$ the von Neumann algebra of the unimodular random network $\mu$. Since $F\left(C\left(\mathcal{G}_{\star}(S, X)\right) \subset \rho_{\mu}\left(C\left(\mathcal{G}_{\star}(S, X)\right) \rtimes \mathbf{F}_{S}\right)^{\prime}\right.$, we conclude that $R(\mu)=\rho_{\mu}\left(C\left(\mathcal{G}_{\star}(S, X)\right) \rtimes \mathbf{F}_{S}\right)^{\prime \prime} \subset F\left(C\left(\mathcal{G}_{\star}(S, X)\right)^{\prime}\right.$. Hence, every operator $T \in R(\mu)$ arises from a measurable family $(G, o) \mapsto T_{G, o}$. We extend $\tau_{\mu}$ to a normal, positive linear functional $\operatorname{tr}_{\mu}$ on $R(\mu)$ by the formula

$$
\operatorname{tr}_{\mu}(T):=\left\langle T \delta^{\mu}, \delta^{\mu}\right\rangle=\mathbf{E}\left[\left\langle T_{G, o} \delta_{o}, \delta_{o}\right\rangle\right]:=\int\left\langle T_{G, o} \delta_{o}, \delta_{o}\right\rangle d \mu(G, o) .
$$

The left-regular representation $\lambda_{\mu}: \mathbf{F}_{S} \rightarrow U(\mathscr{H}(\mu))$ is defined as acting on the underlying measure space, i.e., a vector $(G, o) \mapsto \xi(G, o)$ is mapped via $\lambda_{\mu}(w)$ to $(G, o) \mapsto$ $\xi(G, o . w)$. Using similar arguments as above, we see that $F$ (rather than $M$ ) and $\lambda_{\mu}$ combine to give another representation $\lambda_{\mu}: C\left(\mathcal{G}_{\star}(S, X)\right) \rtimes \mathbf{F}_{S} \rightarrow B(\mathscr{H}(\mu))$ and we set $L(\mu):=\lambda_{\mu}\left(C\left(\mathcal{G}_{\star}(S, X)\right) \rtimes \mathbf{F}_{S}\right)^{\prime \prime}$. It is now a matter of checking definitions to see that an operator $T \in B(\mathscr{H}(\mu))$ is equivariant if and only if $T \in L(\mu)^{\prime}$.

Put $N_{\tau_{\mu}}:=\left\{T \in C\left(\mathcal{G}_{\star}(S, X)\right) \rtimes \mathbf{F}_{S} ; \tau_{\mu}\left(T^{*} T\right)=0\right\}$. In order to put the players in the right framework, let us note that $\left(C\left(\mathcal{G}_{\star}(S, X)\right) \rtimes \mathbf{F}_{S}\right) / N_{\tau_{\mu}}$ together with the inner-product $\left(T_{1} \mid T_{2}\right):=\tau_{\mu}\left(T_{2}^{*} T_{1}\right)$ is a Hilbert algebra in the sense of [17, Chapters 5 and 6$]$. The algebras $L(\mu)$ and $R(\mu)$ can be identified with the von Neumann algebras that are left- and rightassociated with this Hilbert algebra. Indeed, as we mentioned above, the associated von Neumann algebras arise from the natural GNS-construction.

It follows from the Commutation Theorem [17, Theorem 1 on page 80] that $R(\mu)=L(\mu)^{\prime}$, i.e., the operators in $R(\mu)$ are precisely the equivariant operators. It was proved by [4] that $T \mapsto \int\left\langle T_{G, o} \delta_{o}, \delta_{o}\right\rangle d \mu(G, o)$ is a trace on the algebra of equivariant operators. This result is also an easy consequence of the general theory of Hilbert algebras; see, for example, [17, Theorem 1 on page 97]. 
Another useful point of view is to see $R(\mu)$ as the von Neumann algebra associated with a discrete measured groupoid; see [51] for a definition. Indeed, consider the $r$-discrete topological groupoid with base space $\mathcal{G}_{\star}(S, X)$ and an arrow between $(G, o)$ and $\left(G^{\prime}, o^{\prime}\right)$ for each $v \in G$ such that $(G, v) \cong\left(G^{\prime}, o^{\prime}\right)$. Any unimodular measure $\mu$ turns this object into a discrete measured groupoid. The von Neumann algebra $R(\mu)$ that has been described concretely above is the von Neumann algebra associated with the discrete measured groupoid associated to the measure $\mu$. We refer to [23] for details about the von Neumann algebra associated to a discrete measured equivalence relation and to [52, Section 3] or [51] for an extension to the realm of discrete measured groupoids.

Let us summarize:

THEOREM 8.3. An operator $T \in B(\mathscr{H}(\mu))$ is equivariant if and only if $T \in R(\mu)$. The pair $\left(R(\mu), \operatorname{tr}_{\mu}\right)$ is a tracial von Neumann algebra.

We illustrate the definitions in two special cases. (1) If $G=(\mathrm{V}, \mathrm{E})$ is a finite $S$-labelled Schreier network with automorphism group $\Lambda$ and we consider the natural action of $\Lambda$ on $\ell^{2} \mathrm{~V}$, then there exists a natural isomorphism

$$
R(U(G)) \stackrel{\sim}{\rightarrow} B\left(\ell^{2} \mathrm{~V}\right)^{\Lambda}:=\left\{T \in B\left(\ell^{2} \mathrm{~V}\right) ; \forall \lambda \in \Lambda T \lambda=\lambda T\right\}
$$

(2) If $\mu$ is concentrated on a Cayley diagram of a group $\Gamma$ (with finite generating set $S$ ) and $X$ is a singleton, then $R(\mu)=R(\Gamma)$.

In complete analogy to the group case, we can define the von Neumann algebra $R(\mu, S) \subset$ $B(\mathscr{H}(\mu, S))$, where

$$
\mathscr{H}(\mu, S):=\int_{\mathcal{G}_{\star}(S, X)} \ell^{2}(\mathrm{E}(G)) d \mu(G, o) .
$$

Again, there is a natural isomorphism $R(\mu, S)=M_{S}(R(\mu))$. We denote the natural trace on $R(\mu, S)$ by $\operatorname{tr}_{\mu}: R(\mu, S) \rightarrow \mathbb{C}$.

We shall now state and prove an embedding theorem for sofic unimodular networks. The techniques are inspired by work of Elek and Lippner [22] and Păunescu [47]. Again, the point is not to give another proof of these results, but to prove new approximation formulas that allow for applications to the approximation of the associated determinantal measures.

THEOREM 8.4. Let $\left(G_{n}\right)_{n}$ be a sequence of finite S-labelled Schreier networks and let $\mu$ be a probability measure on $\mathcal{G}_{\star}(S, X)$. Let $\omega$ be a non-principal ultrafilter on $\mathbb{N}$. If $U\left(G_{n}\right) \Rightarrow \mu$, then there exists a trace-preserving embedding

$$
\iota:\left(R(\mu), \operatorname{tr}_{\mu}\right) \rightarrow \prod_{n \rightarrow \omega}\left(R\left(U\left(G_{n}\right)\right), \operatorname{tr}_{U\left(G_{n}\right)}\right) .
$$

Moreover, there exists a sequence of probability measures $\left(\nu_{n}\right)_{n}$ on $\left(\left\{G_{n}\right\} \times \mathrm{V}\left(G_{n}\right)\right) \times \mathcal{G}_{\star}(S, X)$ with the following properties: 
(i) The measure $\nu_{n}$ has marginals $U\left(G_{n}\right)$ and $\mu$.

(ii) With respect to the natural metric $d_{*}$ on $\mathcal{G}_{\star}(S, X)$, we have

$$
\lim _{n \rightarrow \infty} \int d_{*}\left(\left(G_{n}, v\right),(G, o)\right) d \nu_{n}\left(\left(G_{n}, v\right),(G, o)\right) \rightarrow 0 .
$$

(iii) If $\left(T_{n}\right)_{n} \in \ell^{\infty}\left(\mathbb{N},\left(R\left(U\left(G_{n}\right)\right), \operatorname{tr}_{U\left(G_{n}\right)}\right)\right)$ represents $\iota(T)$ for some $T \in R(\mu)$, then

$$
\lim _{n \rightarrow \omega} \int\left|\left\langle T_{n} \delta_{v \cdot \gamma}, \delta_{v \cdot \gamma^{\prime}}\right\rangle-\left\langle T_{G, o} \delta_{o . \gamma}, \delta_{o . \gamma^{\prime}}\right\rangle\right| d \nu_{n}\left(\left(G_{n}, v\right),(G, o)\right)=0
$$

for all $\gamma, \gamma^{\prime} \in \mathbf{F}_{S}$.

Proof. First of all, $\mu$ is unimodular. Recall the positive and unital trace $\tau_{\mu}: C\left(\mathcal{G}_{\star}(S, X)\right) \rtimes$ $\mathbf{F}_{S} \rightarrow \mathbb{C}$ defined in (7). As in the proof of Proposition 4.4, we consider the unital $*$ homomorphism

$$
\rho:=\lim _{n \rightarrow \omega} \rho_{U\left(G_{n}\right)}: C\left(\mathcal{G}_{\star}(S, X)\right) \rtimes \mathbf{F}_{S} \rightarrow \prod_{n \rightarrow \omega}\left(R\left(U\left(G_{n}\right)\right), \operatorname{tr}_{U\left(G_{n}\right)}\right) .
$$

Since $U\left(G_{n}\right) \Rightarrow \mu$, we have that $\tau_{\mu}=\operatorname{tr}_{\omega} \circ \rho$, where $\operatorname{tr}_{\omega}$ denotes the trace on the ultraproduct, i.e., $\operatorname{tr}_{\omega}\left(\left(T_{n}\right)_{n}\right):=\lim _{n \rightarrow \omega} \operatorname{tr}_{U\left(G_{n}\right)}\left(T_{n}\right)$ for all norm-bounded sequences $\left(T_{n}\right)_{n}$ with $T_{n} \in R\left(U\left(G_{n}\right)\right)$. As $\operatorname{tr}_{\mu}$ is faithful on $R(\mu), \rho$ factors through the image $\operatorname{im}\left(\rho_{\mu}\right)$ of $\rho_{\mu}: C\left(\mathcal{G}_{\star}(S, X)\right) \rtimes \mathbf{F}_{S} \rightarrow R(\mu)$. That is, there is a unique bounded $*$-homomorphism

$$
\psi: \operatorname{im}\left(\rho_{\mu}\right) \rightarrow \prod_{n \rightarrow \omega}\left(R\left(U\left(G_{n}\right)\right), \operatorname{tr}_{U\left(G_{n}\right)}\right)
$$

such that $\rho=\psi \circ \rho_{\mu}$. By definition $\operatorname{im}\left(\rho_{\mu}\right)$ is weakly dense in $R(\mu)$, whence $\psi$ extends to a trace-preserving $*$-homomorphism $\iota$ from $R(\mu)$ to the ultraproduct von Neumann algebra.

Weak convergence of measures on $\left(\mathcal{G}_{\star}(S, X), d_{*}\right)$ is equivalent (see the last corollary in $[55]$ or $[53,3.1 .1])$ to convergence in the Wasserstein metric

$$
d_{\mathrm{W}}\left(\mu^{\prime}, \mu\right):=\inf _{\nu} \int d_{*}\left(\left(G^{\prime}, o^{\prime}\right),(G, o)\right) d \nu\left(\left(G^{\prime}, o^{\prime}\right),(G, o)\right),
$$

where the infimum is taken over all measures $\nu$ on $\mathcal{G}_{\star}(S, X) \times \mathcal{G}_{\star}(S, X)$ with marginals $\mu^{\prime}$ and $\mu$. Hence, we obtain a sequence of measures $\nu_{n}^{\prime}$ on $\mathcal{G}_{\star}(S, X) \times \mathcal{G}_{\star}(S, X)$ with marginals $U\left(G_{n}\right)$ and $\mu$ so that

$$
\lim _{n \rightarrow \infty} \int d_{*}\left(\left(G^{\prime}, o^{\prime}\right),(G, o)\right) d \nu_{n}^{\prime}\left(\left(G^{\prime}, o^{\prime}\right),(G, o)\right) \rightarrow 0 .
$$

Since the natural map $\left(G_{n} \times \mathrm{V}\left(G_{n}\right)\right) \times \mathcal{G}_{\star}(S, X) \rightarrow \mathcal{G}_{\star}(S, X) \times \mathcal{G}_{\star}(S, X)$ is finite-to-one, we can lift $\nu_{n}^{\prime}$ to a measure $\nu_{n}$ on $\left(G_{n} \times \mathrm{V}\left(G_{n}\right)\right) \times \mathcal{G}_{\star}(S, X)$. This proves (i) and (ii).

It remains to prove claim (iii) and in doing so, we follow the strategy of the proof of Proposition 4.4. Indeed, using the arguments in the proof of Proposition 4.4, it is again easy to see that the truth of (iii) depends only on $T \in R(\mu)$ and not on the choice of 
an approximating sequence $\left(T_{n}\right)_{n}$. If $T$ lies in the image of $C\left(\mathcal{G}_{\star}(S, X)\right) \rtimes \mathbf{F}_{S}$ and $T^{\prime}=$ $\sum_{w} f_{w} w$ is some choice of a preimage of $T$ in the crossed product algebra, then there is a canonical approximating sequence $\left(T_{n}\right)_{n}$ that represents $\iota(T)$. Indeed, for each $n$, there is a $*$-homomorphism $\rho_{U\left(G_{n}\right)}: C\left(\mathcal{G}_{\star}(S, X)\right) \rtimes \mathbf{F}_{S} \rightarrow R\left(U\left(G_{n}\right)\right)$ and we set $T_{n}:=\rho_{U\left(G_{n}\right)}\left(T^{\prime}\right)$ for each $n \in \mathbb{N}$. For each $w \in \mathbf{F}_{S}$, the function $f_{w}$ in the presentation of $T^{\prime}$ is uniformly continuous and hence $(G, o) \mapsto\left\langle T \delta_{o . \gamma}, \delta_{o . \gamma^{\prime}}\right\rangle$ is uniformly continuous as well for such $T$. Thus, (ii) easily implies (iii) for every element in the image of $C\left(\mathcal{G}_{\star}(S, X)\right) \rtimes \mathbf{F}_{S}$. As in the proof of Proposition 4.4, a diagonalization argument shows that (iii) holds for all $T \in R(\mu)$. This finishes the proof.

\section{Existence of sofic monotone couplings}

Let $\mu$ be a unimodular probability measure on rooted networks. Given an equivariant positive contraction $Q$ on $\mathscr{H}(\mu)$, we obtain a determinantal probability measure $\mathbf{P}^{Q_{G}}$ on $\{0,1\}^{\mathrm{V}(G)}$ associated to $\mu$-a.e. rooted network $(G, o)$. Note that if $G$ already has marks, then we regard $\mathbf{P}^{Q_{G}}$ as producing (at random) new marks, $\eta \in\{0,1\}^{\mathrm{V}(G)}$, which we may

take formally as second coordinates after the existing marks. In other words, we define the probability measure $\mu^{Q}$ by the equation

$$
\mu^{Q}\left[B(o, r ; G) \cong(A, v), \eta\lceil C \equiv 1]=\int_{[B(o, r ; G) \cong(A, v)]} \operatorname{det}\left(Q_{G}\lceil C) d \mu(G, o)\right.\right.
$$

for every rooted network $(A, v)$ of radius $r$ and every measurable choice of $C \subseteq B(o, r ; G)$. Using involution invariance, it is easy to check that $\mu^{Q}$ is unimodular.

As shown in Proposition 8.2, we may assume that $\mu$ is carried by $S$-labelled Schreier networks. In this case, it suffices to take the measurable choice of $C \subseteq B(o, r ; G)$ in the definition of $\mu^{Q}$ to be of the form $\left\{o . w_{1}, \ldots, o . w_{n}\right\}$ for some $w_{1}, \ldots, w_{n} \in \mathbf{F}_{S}$.

As a special case, let $G$ be a finite network and $Q$ be a positive contraction on $\ell^{2}(\mathrm{~V}(G))$. Then $U(G)^{Q}$ is the determinantal probability measure reviewed in Section 2, regarded as a randomly rooted network.

Given a unimodular probability measure $\mu$ with mark space $\Xi$ and two unimodular probability measures $\mu_{i}$ with mark spaces $\Xi \times\{0,1\}$ for $i=1,2$, both of whose marginals forgetting the second coordinate of the marks is $\mu$, we say that a unimodular probability measure $\nu$ with the 3 -coordinate mark space $\Xi \times\{0,1\} \times\{0,1\}$ is a monotone coupling of $\mu_{1}$ and $\mu_{2}$ if its marginal forgetting the coordinate $4-i$ is $\mu_{i}$ for $i=1,2$ and $\nu$ is concentrated on networks whose marks $(\xi, j, k)$ satisfy $j \leq k$.

We shall prove the following extension of Theorem 5.1: 
THEOREM 9.1. Let $\mu$ be a sofic probability measure on rooted networks. If $0 \leq Q_{1} \leq$ $Q_{2} \leq I$ in $R(\mu)$, then there exists a sofic monotone coupling of $\mathbf{P}^{Q_{1}}$ and $\mathbf{P}^{Q_{2}}$.

As we noted, we may assume that $\mu$ is carried by Schreier networks. We have the following extension of Lemma 4.5:

LEMma 9.2. Let $\left(G_{n}\right)_{n}$ be a sequence of finite S-labelled Schreier networks whose random weak limit is $\mu$. Let $\iota$ and $\iota_{S}$ be trace-preserving embeddings as in Theorem 8.4. Let $T \in$ $R(\mu)$ be such that $0 \leq T \leq I$ and suppose that $\left(T_{n}\right)_{n}$ represents $\iota(T)$ in the ultraproduct $\prod_{n \rightarrow \omega}\left(B\left(\ell^{2} \bigvee_{n}\right), \operatorname{tr}_{\vee_{n}}\right)$ with $0 \leq T_{n} \leq I$ for each $n \in \mathbb{N}$. Then $\lim _{n \rightarrow \omega} U\left(G_{n}\right)^{T_{n}}=\mu^{T}$ in the weak topology.

The proof is essentially the same as that for Lemma 4.5, using the coupling probability measures $\nu_{n}$ of Theorem 8.4 (but not assuming any analogue of the injectivity of $\pi$ ).

We may now use Lemma 9.2 to prove Theorem 9.1, just as Theorem 5.1 was proved.

All the above was for determinantal probability measures on subsets of vertices. In order to deduce corresponding results for determinantal probability measures on subsets of edges (or even "mixed" measures on subsets of both vertices and edges), we use the following construction. Given a network $G$, subdivide each edge $e$ by adding a new vertex $x_{e}$, which is joined to each endpoint of $e$ and which receives the mark of $e$. Also assign a second coordinate to the new vertices so that we may distinguish them. Provided that the expected degree of the root under the unimodular measure $\mu$ is finite, we may choose a re-rooting of the subdivided networks in order to obtain a natural unimodular probability measure, $\tilde{\mu}$ : see [4, Example 9.8] for details. In fact, when $\mu$ is the random weak limit of $\left(G_{n}\right)_{n}$, we may simply subdivide the edges of $G_{n}$ and take the random weak limit of the resulting networks, $G_{n}^{\prime}$. Using the finiteness of the expected degree under $\mu$, it is not hard to check that $U\left(G_{n}^{\prime}\right)$ does indeed converge to $\tilde{\mu}$. With this construction, if we desire a determinantal probability measure on the edges for $\mu$, we may simply use the corresponding positive contraction on the vertices for $\tilde{\mu}$, where all entries are 0 that do not correspond to a pair of new vertices.

In particular, this result allows to extend our observations on the existence of invariant monotone couplings between WSF and FSF to unimodular random networks. In combination with the results in [2], we are also able to extend the results in Section 6. We omit proofs since the strategy and the techniques are unchanged.

\section{Acknowledgments}

This research started when R.L. visited Universität Göttingen as a guest of the Courant Research Centre Göttingen in November 2008 and was continued later when A.T. visited Indiana University at Bloomington as a Visiting Scholar in March 2011. A.T. thanks ERC for support. We thank Ben Hayes for help with the proof of Lemma 7.9. 


\section{References}

[1] Abért, M. and Weiss, B., Bernoulli actions are weakly contained in any free action. Ergodic Theory Dynam. Systems, 33(2) (2013), 323-333.

[2] Abért, M., Virág, B. and Thom, A., Benjamini-Schramm convergence and pointwise convergence of the spectral measure. Preprint, (2011).

[3] Adams, S. , Very weak Bernoulli for amenable groups. Israel J. Math., 78(2-3), (1992) 145-176.

[4] Aldous, D.J. and Lyons, R., Processes on unimodular random networks. Electron. J. Probab. 12 (2007), no. 54, 1454-1508 (electronic).

[5] Bekka, M., Valette, A., Group cohomology, harmonic functions and the first $L^{2}$-Betti number. Potential Anal. 6(4), (1997) 313-326.

[6] Benjamini, I., Lyons, R., Peres, Y., and Schramm, O., Uniform spanning forests. Ann. Probab. 29, (2001) 1-65.

[7] Benjamini, I. and Schramm, O., Recurrence of distributional limits of finite planar graphs. Electron. J. Probab. 6 (2001b), no. 23, 13 pp. (electronic).

[8] Borcea, J., Brändén, P., and Liggett, T.M., Negative dependence and the geometry of polynomials. J. Amer. Math. Soc. 22 (2009), 521-567.

[9] Bowen, L., Couplings of uniform spanning forests. Proc. Amer. Math. Soc. 132 (2004), no. 7, 2151-2158.

[10] Bowen, L., Measure conjugacy invariants for actions of countable sofic groups. J. Amer. Math. Soc. 23 (2010), no. 1, 217-245.

[11] Brooks, R.L., Smith, C.A.B., Stone, A.H., and Tutte, W.T., The dissection of rectangles into squares. Duke Math. J. 7 (1940), 312-340.

[12] Brown, N. and Ozawa, N., $C^{*}$-Algebras and Finite-Dimensional Approximations. Cambridge U. Press, 1994.

[13] Burton, R.M. and Pemantle, R., Local characteristics, entropy and limit theorems for spanning trees and domino tilings via transfer-impedances. Ann. Probab. 21 (1993), 1329-1371.

[14] Chifan, I. and Ioana, A., Ergodic subequivalence relations induced by a Bernoulli action. Geom. Funct. Anal., 20(1) (2010), 53-67.

[15] Connes, A., Classification of injective factors cases $I I_{1}, I I_{\infty}, I I I_{\lambda}, \lambda \neq 1$. Ann. of Math., 2nd Series, 104, No. 1 (1976), 73-115.

[16] Cornulier, Y., A sofic group away from amenable groups. Math. Ann. 350:2 (2011), 269-275.

[17] Dixmier, J., Von Neumann Algebras, North-Holland Mathematical Library 27, Amsterdam (1981), xxxviii +437 .

[18] Douglas, R.G., On majorization, factorization, and range inclusion of operators on Hilbert space. Proc. Amer. Math. Soc. 17, No. 2 (1966), 413-415.

[19] Elek, G. and Szabó, E., Sofic groups and direct finiteness. J. Algebra 280 (2004), no. 2, 426-434.

[20] Elek, G. and Szabó, E., Hyperlinearity, essentially free actions and $L^{2}$-invariants. The sofic property. Math. Ann. 332:2 (2005), 421-441.

[21] Elek, G. and Szabó, E., On sofic groups. J. of Group Theory 9:2 (2006), 161-171.

[22] Elek, G. and Lippner, G., Sofic equivalence relations, J. Funct. Anal. 258:5 (2010), 1692-1708.

[23] Feldman, J. and Moore, C., Ergodic equivalence relations, cohomology, and von Neumann algebras. II Trans. Amer. Math. Soc. 234 (2) (1977) 325-359. 
[24] Fontes, L.R.G. and Mathieu, P., On symmetric random walks with random conductances on $\mathbb{Z}^{d}$. Probab. Theory Related Fields 134 (2006), 565-602.

[25] Glasner, E., Ergodic Theory via Joinings. American Mathematical Society, Providence, RI. (2003).

[26] Häggström, O., Random-cluster measures and uniform spanning trees. Stochastic Process. Appl. 59 (1995), 267-275.

[27] Houdayer, C., Invariant percolation and measured theory of nonamenable groups [after GaboriauLyons, Ioana, Epstein]. Astérisque, 348 (2012), Exp. No. 1039, ix, 339-374. Séminaire Bourbaki: Vol. 2010/2011. Exposés 1027-1042.

[28] Kadison, R.V. and Ringrose, J.R., Fundamentals of the Theory of Operator Algebras. Vol. I, volume 15 of Graduate Studies in Mathematics. American Mathematical Society, Providence, RI. (1997). Elementary theory, Reprint of the 1983 original.

[29] Kadison, R.V. and Ringrose, J.R., Fundamentals of the Theory of Operator Algebras. Vol. II, volume 16 of Graduate Studies in Mathematics. American Mathematical Society, Providence, RI. (1997). Advanced theory, Corrected reprint of the 1986 original.

[30] Kulesza, A. and Taskar, B., Determinantal point processes for machine learning, Foundations and Trends in Machine Learning, 5, 2-3 (2012), 123-286. DOI: 10.1561/2200000044.

[31] Kechris, A., Solecki, S. and Todorčević, S., Borel chromatic numbers, Advances in Mathematics 141 (1999), 1-44.

[32] Kirchhoff, G., Ueber die Aufösung der Gleichungen, auf welche man bei der Untersuchung der linearen Vertheilung galvanischer Ströme geführt wird. Ann. Phys. und Chem. 72 (1847), 497-508.

[33] Kun, G., Expanders have a spanning Lipschitz subgraph with large girth. Preprint, http: //www.arxiv . org/abs/1303.4982, (2013).

[34] Lück, W., Approximating $L^{2}$-invariants by their finite-dimensional analogues. Geom. Funct. Analysis 4 (1994) 455-481.

[35] Lück, W., L2-Invariants: Theory and Applications to Geometry and K-Theory. Ergebnisse der Mathematik und ihrer Grenzgebiete, 3. A Series of Modern Surveys in Mathematics, 44. Springer, Berlin, 2002.

[36] Lyons, R., A bird's-eye view of uniform spanning trees and forests. In Aldous, D. and Propp, J., editors, Microsurveys in Discrete Probability, volume 41 of DIMACS Series in Discrete Mathematics and Theoretical Computer Science, pages 135-162. Amer. Math. Soc., Providence, RI. (1998). Papers from the workshop held as part of the Dimacs Special Year on Discrete Probability in Princeton, NJ, June 2-6, 1997.

[37] Lyons, R., Determinantal probability measures. Publ. Math. Inst. Hautes Études Sci. 98 (2003), 167212. Errata, http://mypage.iu.edu/ rdlyons/errata/bases.pdf.

[38] Lyons, R. and Steif, J.E., Stationary determinantal processes: Phase multiplicity, Bernoullicity, entropy, and domination. Duke Math. J., 120(3) (2003), 515-575.

[39] Lyons, R., Fixed price of groups and percolation. Ergodic Theory Dynam. Systems, 33(1) (2013), 183185.

[40] Lyons, R., Factors of IID on trees. Preprint, http://www.arxiv.org/abs/1401.4197, (2013).

[41] Macchi, O., The coincidence approach to stochastic point processes, Advances in Appl. Probability 7 (1975), 83-122.

[42] Mester, P., Invariant monotone coupling need not exist. Ann. Probab. 41, 3A (2013), 1180-1190. 
[43] Nielsen, O.A., Direct Integral Theory, volume 61 of Lecture Notes in Pure and Applied Mathematics. Marcel Dekker Inc., New York. (1980).

[44] Ornstein, D. , Factors of Bernoulli shifts are Bernoulli shifts. Advances in Math., 5, (1970) 349-364.

[45] Ornstein, D.S., Ergodic Theory, Randomness, and Dynamical Systems. Yale University Press, New Haven, Conn., 1974. James K. Whittemore Lectures in Mathematics given at Yale University, Yale Mathematical Monographs, No. 5.

[46] Ornstein, D.S. and Weiss, B., Entropy and isomorphism theorems for actions of amenable groups. $J$. Analyse Math., 48, (1987) 1-141.

[47] Păunescu, L., On sofic actions and equivalence relations J. Funct. Anal. 261:9 (2011), 2461-2485.

[48] Pemantle, R., Choosing a spanning tree for the integer lattice uniformly. Ann. Probab. 19 (1991), $1559-1574$.

[49] Peterson, J. and Thom, A., Group cocycles and the ring of affiliated operators. Invent. Math. 185 (2011), 561-592.

[50] Popa, S., Some computations of 1-cohomology groups and construction of non-orbit-equivalent actions. J. Inst. Math. Jussieu, 5(2), (2006) 309-332.

[51] Renault, J.N., A Groupoid Approach to $C^{*}$-algebras, Lecture Notes in Math., no. 793, Springer-Verlag, 1980.

[52] Sauer, R., $L^{2}$-Betti numbers of discrete measured groupoids, Int. J. Algebra and Computation 15, 5 \& 6 (2005), 1169-1188.

[53] Skorohod, A.V., Limit theorems for stochastic processes. Teor. Veroyatnost. i Primenen. 1 (1956), 289-319. In Russian. English translation in Theory Probab. Appl. 1 (1956), 261-290.

[54] Soshnikov, A., Determinantal random point fields, Uspekhi Mat. Nauk 55 (2000), 107-160.

[55] Strassen, V., The existence of probability measures with given marginals. Ann. Math. Statist. 36 (1965), 423-439.

[56] Takesaki, M., Theory of Operator Algebras. III. Encyclopaedia of Mathematical Sciences, 127. Operator Algebras and Non-Commutative Geometry, 8. Springer, Berlin, 2003.

[57] Thom, A., Sofic groups and Diophantine approximation. Comm. Pure Appl. Math., 61(8) (2008), 11551171.

[58] Timár, Á., Ends in free minimal spanning forests. Ann. Probab. 34 (2006), 865-869.

[59] Weiss, B., Sofic groups and dynamical systems. Ergodic theory and harmonic analysis (Mumbai, 1999). Sankhya Ser. A 62 (2000), no. 3, 350-359.

R.L., Dept. of Math., 831 E. 3Rd St., Indiana Univ., Bloomington, IN 47405-7106, USA

E-mail address: rdlyons@indiana.edu

A.T., Math. Institut, Univ. Leipzig, PF 100920, D-04009 Leipzig, Germany

E-mail address: thom@math.uni-leipzig.de 